\title{
Modeling of planar plasma diode
}

\author{
K. A. I. L. Wijewardena Gamalath, A. M. Samarakoon \\ University of Colombo, Colombo 3, Sri Lanka \\ Email address: imalie@phys.cmb.ac.lk
}

\begin{abstract}
To investigate the dynamics of a planar plasma diode system (PDS), a model based on the current density equilibrium at the interface was developed. The current densities and plasma boundary variations with the potential fields were obtained by simulating a single square pulse. The variation of an observed overshoot current density with the applied voltage is presented. Planar plasma diode system was also simulated for periodic, sine, square, triangular and saw tooth voltage patterns by varying the amplitude and frequencies. A method to find the lower bound of the electron density of plasma for a specified PDS is presented. Particle-In-Cell simulation technique was used to investigate the plasma particles and electric field distributions over the anode cathode gap for different intensities of external electric fields. The system became stable after few time steps and this time depends upon the intensity and polarization of the external field.
\end{abstract}

Keywords: Plasma diode; plasma boundary; current density; Particle-In-Cell simulation; anode cathode gap; potential field

\section{INTRODUCTION}

During the last decades, great attention was directed to plasma formation by ferroelectric emission [1] whose cathodes operate with high repetition rate producing uniform electron beams [2]. The electron emission from the surface plasma as a result of incomplete discharge along the surface of the ferroelectric sample [3] or electron avalanching along the tangential component of the applied electric field is enhanced due to the high dielectric constant of ferroelectrics [4]. In addition, the observed surface discharge is accompanied by a neutral flow that causes vacuum deterioration [5] and due to its expansion along the surface of the ferroelectric, the plasma serves as a dynamic front electrode. Oscillatory nonlinear electron flow in a Pierce diode was studied by Godfrey [6] to obtain a static solution and an integral formulation was presented by Schamel and Maslov [7] taking into account the ion dynamics. Nonlinear dynamics of a plasma filled diode in the presence of a magnetic field was investigated by Li et.at [8] under steady, oscillatory, chaotic and unstable dynamical regimes.

A planar diode with plasma cathode in one dimension was modeled by considering equilibrium current densities at the plasma boundary using Runge-Kutta-Fehlberg method with adaptive step size. By means of the model, the current densities and plasma boundary variation with the potential field was tested for ranges of A-C gap of the diode, the electron density of the plasma and thermal energy of plasma electrons for a single square voltage pulse varying its amplitude over, delay time, duration, and rise/fall time of the pulse. The current density through the diode and the plasma boundary with system parameters and square pulse 
parameters are presented. As an overshoot at rising edge of the current density was noticed, the relationship between overshoot current density and the applied voltage was obtained. Plasma diode system was simulated for sine, square, triangular and saw tooth periodic voltage patterns by varying the amplitude over (0-10) $\mathrm{kV}$ and frequency over (0-2000) $\mathrm{MHz}$.

The plasma boundary depends only on the amplitude of the applied pulse, electron density of plasma and the A-C gap, and a lower bound of the electron density can be introduced for a specified system and a method to find it for a specified PDS, is presented. Particle-In-Cell simulation technique was used to investigate the plasma particles and the electric field distribution over the anode cathode gap for different intensities of external electric field $0-2 \times 10^{-25} \mathrm{Vm}$ and the number density of electrons $\sim 2000 \mathrm{~mm}^{-2}$. The plasma diode system became stable with time and the time taken to make the system stabilized, depended on the strength of applied electric field.

\section{THE DYNAMICS OF THE CHARGED PARTICLE}

Plasma, an ionized state of matter differs from a neutral gas due to different character of inter-particle forces in them. In a neutral gas, this short range and strong force is of Van der Waals' type whereas in plasma, this force is of the Coulomb type that is long range and weak at very large distances. Since each particle of the plasma interacts in many ways, it has very complex dynamics and physics is rich with information. Plasma is electrically neutral or quasi-neutral but on the scale of the Debye length $\left(\lambda_{D}\right)$, there can be charge imbalance, since the Coulomb force among the plasma particles varies as inverse square of the distance. The effective electric field of the charge does not extend to infinity, but to a characteristic distance, Debye length $\lambda_{D}=\sqrt{\varepsilon_{0} k T / n_{0} e^{2}}$ given in terms of the charge density $\rho_{0}$, the number density of the plasma $n_{0}$, the permittivity of the vacuum $\varepsilon_{0}$, the electron charge $e$, mass $m_{e}$, the Boltzmann constant $k$ and the temperature of the electrons $T$ while the plasma frequency $\omega_{p}$ is $\sqrt{e \rho_{0} / \varepsilon_{0} m_{e}}$. The thermal velocity $v_{t h_{i}}$ of electrons and ions are $\sqrt{8 k T_{i} / \pi m_{i}}$. For a $L$ dimension plasma with $n_{e}$ number density of electrons and collision frequency $v$, the important conditions of the plasma are given by $L \gg \lambda_{D} \gg n_{e}^{-1 / 3}$ and $v<\omega_{p}$. The particle flux given in terms of the coefficient of diffusion $D$ is $\Gamma=-D \nabla n$. The equation of motion of a plasma particle with a drift velocity $\boldsymbol{v}$, including collisions is given by,

$m n \frac{d \boldsymbol{v}}{d t}= \pm e n \boldsymbol{E}-\nabla P-m n v \boldsymbol{v} \quad(\boldsymbol{v}=\mu \boldsymbol{E})$

where $\mu$ is the mobility of electrons or ions, $P$ is the pressure, $\boldsymbol{E}$ is the electric field and the sign \pm indicate the sign of the charge. For isothermal plasma, the pressure is $n k T$. The dynamics of plasma particles is governed by electromagnetic fields and Maxwell's equations lead to continuity equation. The principle laws, used to model Plasma diode are the ChildLangmuir law and the Bohm law. The plasma saturation current density $\boldsymbol{j}_{e p l} \approx e n_{e} \boldsymbol{v}_{t h}$ is given in terms of electron plasma thermal velocity $v_{t h}$. For an applied voltage $U_{0}$ and ferroelectric sample of dielectric constant $\varepsilon$ and thickness $\delta$, the significant electric field enhancement is $E \approx \varepsilon U_{0} / \delta$. The Child-Langmuir Law, a fairly direct result of the Poisson equation for an 
electron situated in between anode cathode gap, gives the maximum space charge limited current in a planar diode of infinite radius for one-dimensional beam as a function of the length and potential difference between anode and cathode,

$$
j_{e}=\left(\left(4 \varepsilon_{0} / 9\right) \sqrt{2 e / m_{e}}\right) V_{d}^{3 / 2}(t) x^{-2}(t)=2.4 \times 10^{-6} V_{d}^{3 / 2}(t) x^{-2}(t)
$$

where $V_{d}$ is the accelerating voltage and $x$ is the distance between anode and the cathode plasma boundaries. The maximum electron current density that can be extracted from plasma whose electron number density is $n_{e}$ and the thermal velocity $v_{T_{e}}$ and temperature $T_{e}$ is given by the Bohm law,

$$
j_{p l}(t)=e n_{e}\left(v_{T_{e}}+\frac{d x}{d t}\right) .
$$

A plasma system can be implemented through a fluid description and the basic equations governing [9] are the continuity, momentum conservation and the Poisson's equation given by,

$$
\begin{aligned}
& \frac{\partial \rho_{e}}{\partial t}+\frac{\partial\left(\rho_{e} v_{e}\right)}{\partial x}=0 \\
& \frac{\partial v_{e}}{\partial t}+v_{e} \frac{\partial v_{e}}{\partial x}=-\frac{e E}{m_{e}}=\left(\frac{e}{m_{e}}\right) \frac{\partial \phi}{\partial x} \\
& \frac{\partial^{2} \phi}{\partial x^{2}}=\frac{1}{\varepsilon_{0}}\left(\rho_{e}-\rho\right)
\end{aligned}
$$

Where $\varphi, \rho_{e}$ and $v_{e}$ are the electric potential, charge density and the velocity of electrons respectively. The boundary conditions are $\rho(0, t)=\rho_{0}, v_{e}(0, t)=v_{0}, \phi(0, t)=\phi(L, t)=0$ and the initial conditions are given by $\phi(x, 0)$ and $\rho_{e}(x, 0)$ where $x \in[0, L]$.

The geometry of planar plasma diode used to model is shown in the Figure 1. Assuming that the plasma will be formed at the vicinity of the cathode plate, the region between two plates was divided into two parts, the plasma region and the vacuum region with the plasma boundary situated in between the anode-boundary and cathode-boundary gap.

These are the boundary of the plasma determined by the limited current densities of each region, thermal ion velocity of the plasma particles and the voltage of accelerating pulse. Since the ion-mass ratio is very large, the assumptions that the ions in the plasma form an immobile charge background and electrons are the only carriers contributing to the current densities in each region were made. 


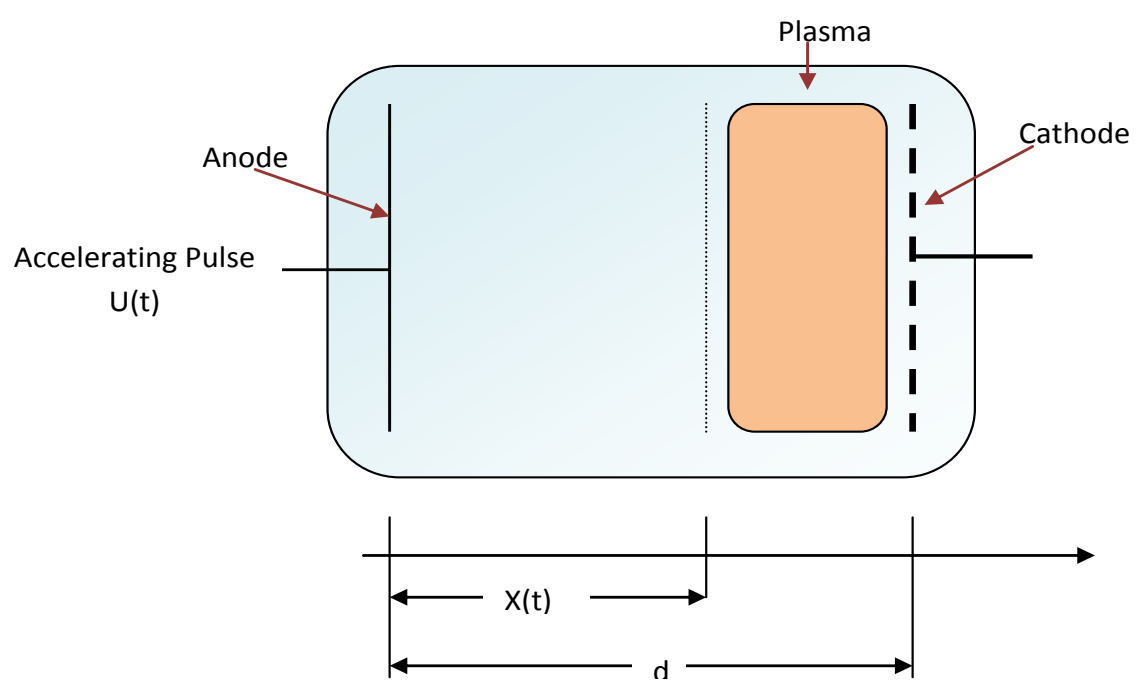

Fig. 1. The geometry of plasma diode used to model.

Therefore vacuum current density and plasma current density are governed by the Child-Langmuir law and Bohm law. According to these laws, the current density of the plasma diode is limited and they are the space charge limit current density $j_{e}$ and the plasma electron saturation current density $j_{p l}$ given in equations 2 and 3 respectively. By applying the Kirchhoff's current law to the plasma boundary $\left(j_{e}=j_{p l}\right)$, the following differential equation,

$$
\frac{d x(t)}{d t}=\frac{4 \varepsilon_{0}}{9 e n_{e}} \sqrt{\frac{2 e}{m_{e}}} \frac{(U(t))^{3 / 2}}{x^{2}(t)}-V_{T_{e}} \quad\left(V_{T_{e}}=\sqrt{\frac{8 k T_{e}}{\pi m_{e}}}\right)
$$

can beobtained. Here $n_{e}$ is the electron plasma density, $T_{e}$ the electron plasma temperature, $d$ the anode cathode gap, $U(t)$ the amplitude of the applied voltage pulse, $x(t)$ the gap between anode and the plasma boundary and $V_{T e}$ is the thermal velocity of electrons.

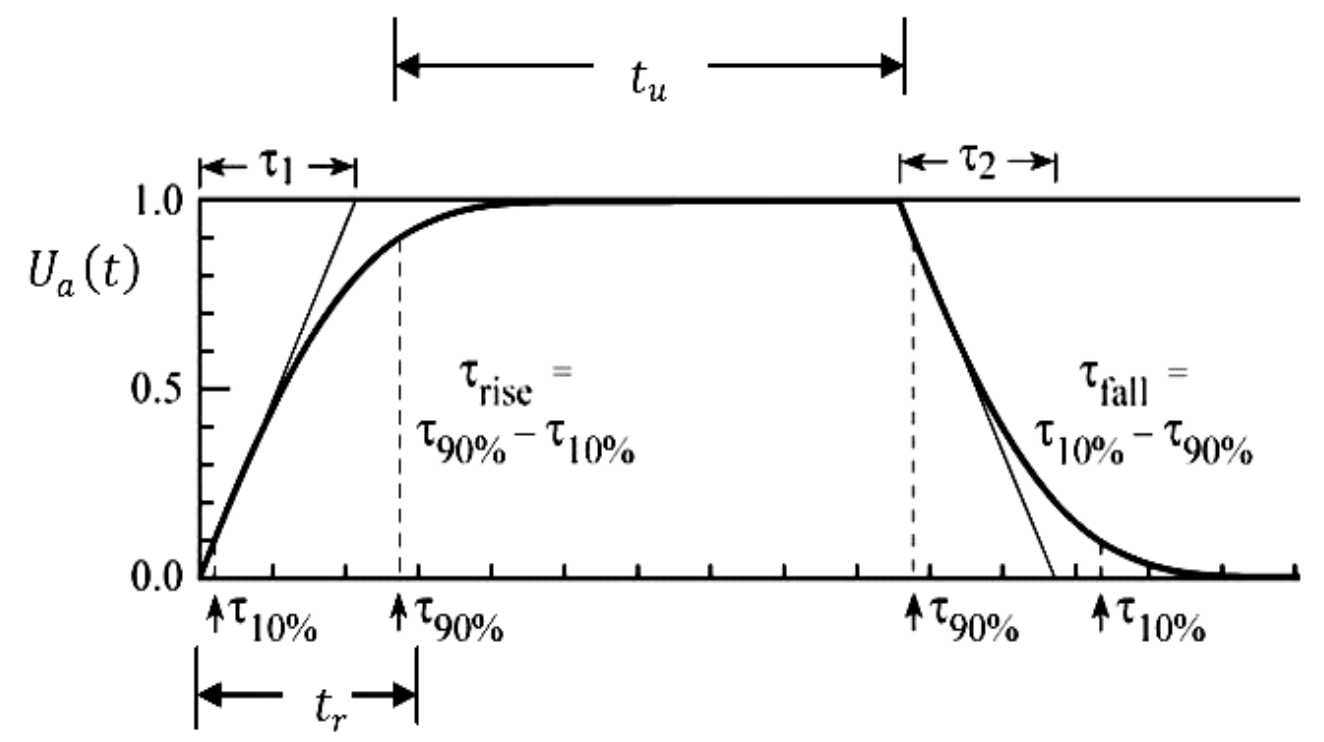

Fig. 2. Parameters of a square pulse. 
This differential equation indicates that the position of plasma boundary depends on the amplitude of the voltage pulse $U_{a}$, the duration of pulse $t_{u}$, the rise/fall time $t_{r}$ and the time delay of the pulse application $\tau$ with respect to initial time $t=0$ (Figure 2). The square wave pulse can be represented as,

$$
U(t)=\begin{array}{cl}
0 & t<\tau \\
\left(U_{a} / t_{r}\right) t & t<\tau+t_{r}
\end{array} \quad U(t)=\begin{array}{cc}
U_{a} & t<\tau+t_{r}+t_{u} \\
U_{a}-\left(U_{a} / t_{r}\right) t & t<\tau+2 t_{r}+t_{u}
\end{array}
$$

Equation 7 was solved using Runge Kutta fifth order method, for the initial conditions $d x / d t_{t=0}=-V_{T_{e}}, x(0)=d$ and ranges of A-C gap of the diode $(0-10) \mathrm{cm}$, the electron density of the plasma $(0-10) \times 10^{21} \mathrm{~m}^{-3}$ and thermal energy of plasma electrons $(0-20) \mathrm{eV}$ simulating for a single square voltage pulse by varying the amplitude over, $(0-10) \mathrm{V}$, delay time $(0-50) n s$, duration $(0-20) n s$, and rise/fall time of the pulse. Input voltage and plasma boundary with respect to time is presented in Figure 3.

Plasma at the vicinity of the cathode began to expand toward the anode with ion thermal velocity until there is non-zero voltage difference between two electrodes. Thereafter the plasma began to erode during the period of non-zero voltage and after the voltage fell to zero, the plasma once again expanded towards the anode. According to the value of time delay of the pulse, the whole A-C gap is filled with the plasma and the diode acts like a perfect conductor.

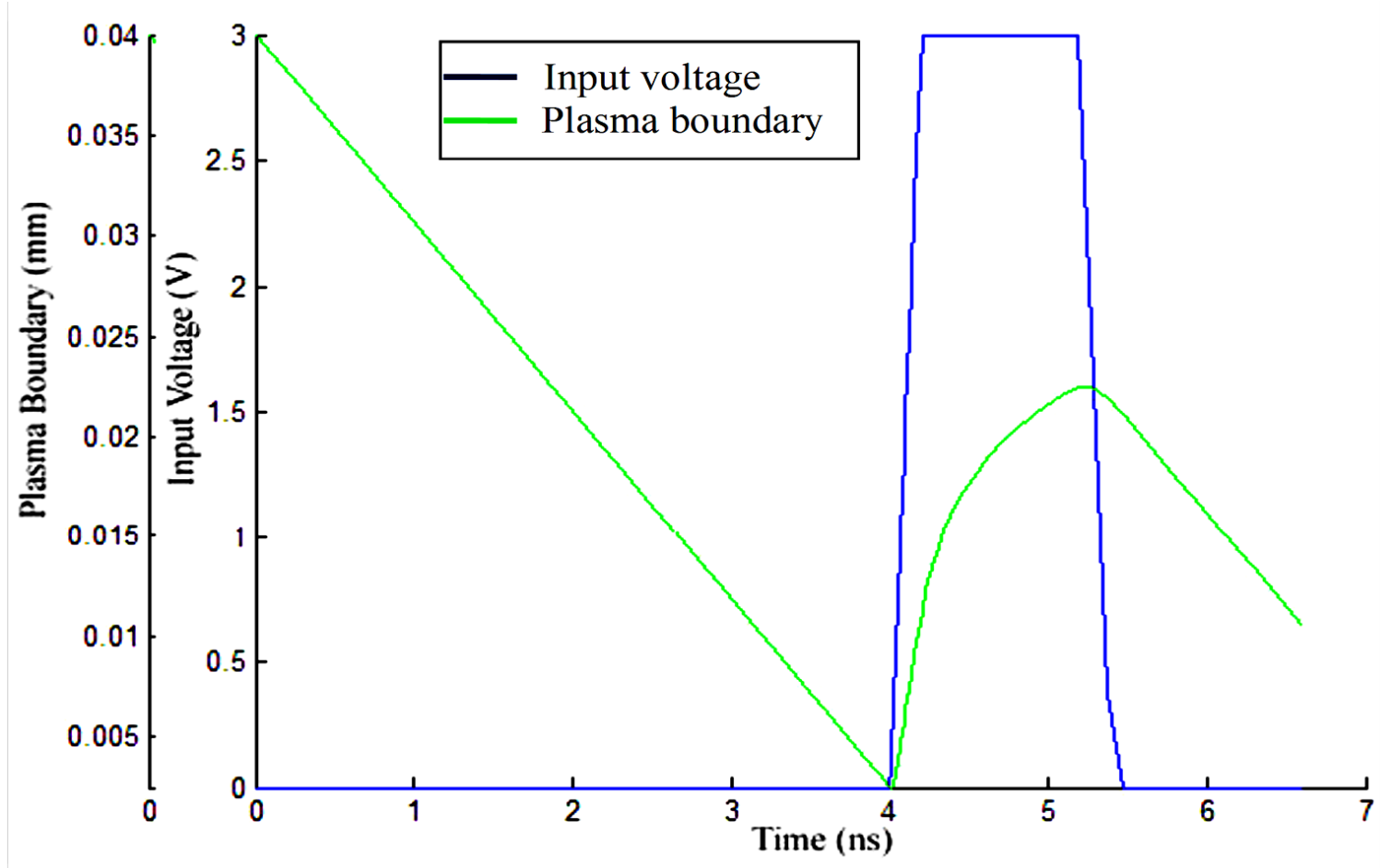

Fig. 3. Input voltage (blue), plasma boundary (green) with time. 
The temporal current density for input voltage pulse of $0.1 \mathrm{~V}$, anode-cathode gap of $4 \mathrm{~cm}$, the electron number density $10^{21} \mathrm{~m}^{-3}$ and electron thermal energy $5 \mathrm{eV}$ is shown in the Figure 4.

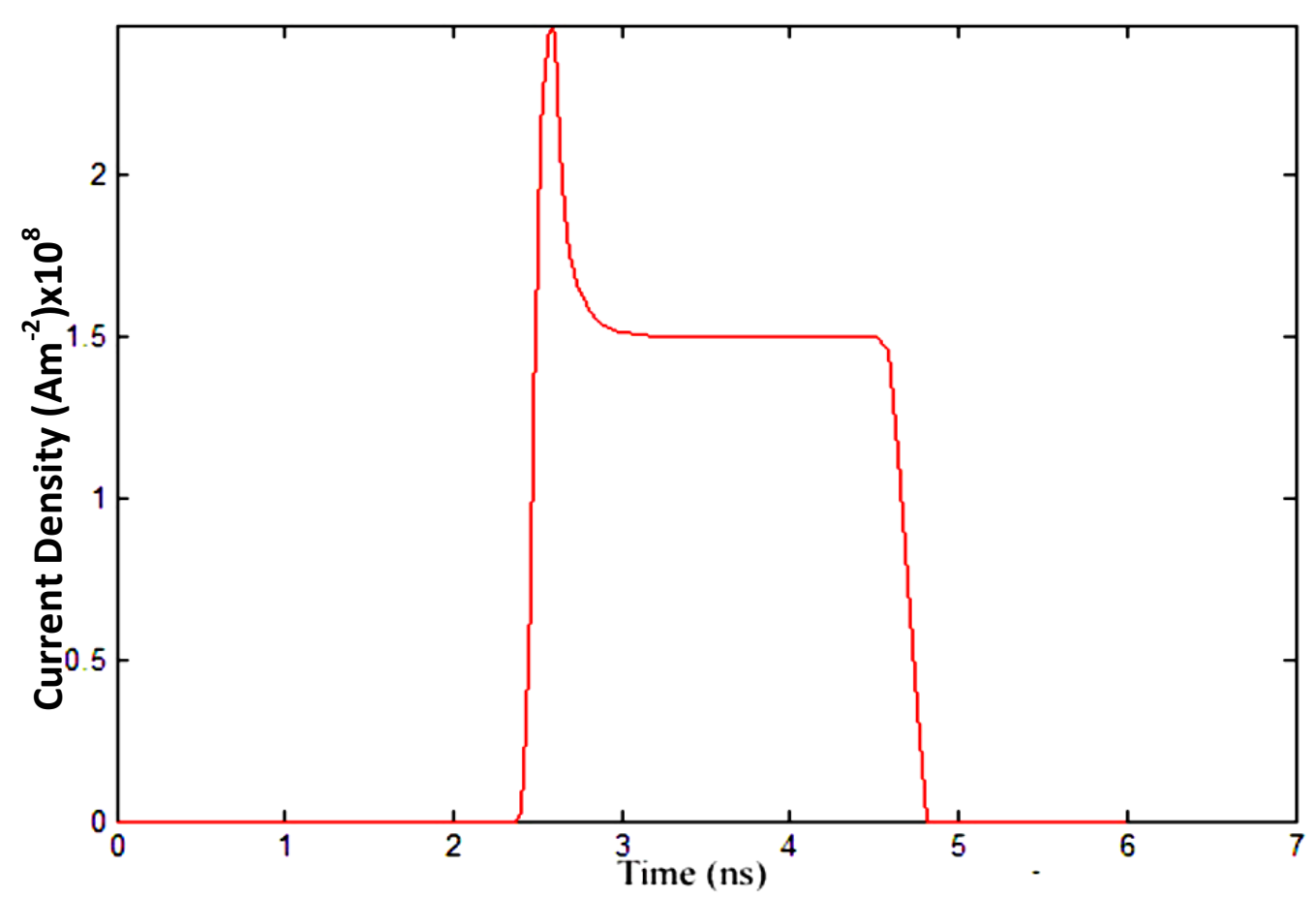

Fig. 4. Current density against time.

The other parameters of the signal pulse used were the same as that of the input pulse of the Figure 3. There is an overshoot at the rising end of the current pulse. Before a voltage was applied to A-C gap, the plasma expanded towards the anode and most of the space in between A-C gap was filled by super conductive plasma.

Thus the effective conductivity between the electrodes was very high compared to a vacuum tube leading to a large current flow with the application of non-zero voltage. But due to the erosion process of the plasma, the effective conductivity between the two electrodes was reduced and the overshoot current density was reduced to a stationary value.

The overshoot and stationary current densities over the amplitude of the input pulse is shown in figure 5 . The relationship between the ratio and the residual of the pulse amplitude, was

$$
\frac{\rho_{s}}{\rho}=\left(-4 \times 10^{-3}\right) \frac{1}{V^{2}}+\left(9.7 \times 10^{-2}\right) \frac{1}{V}+0.19
$$




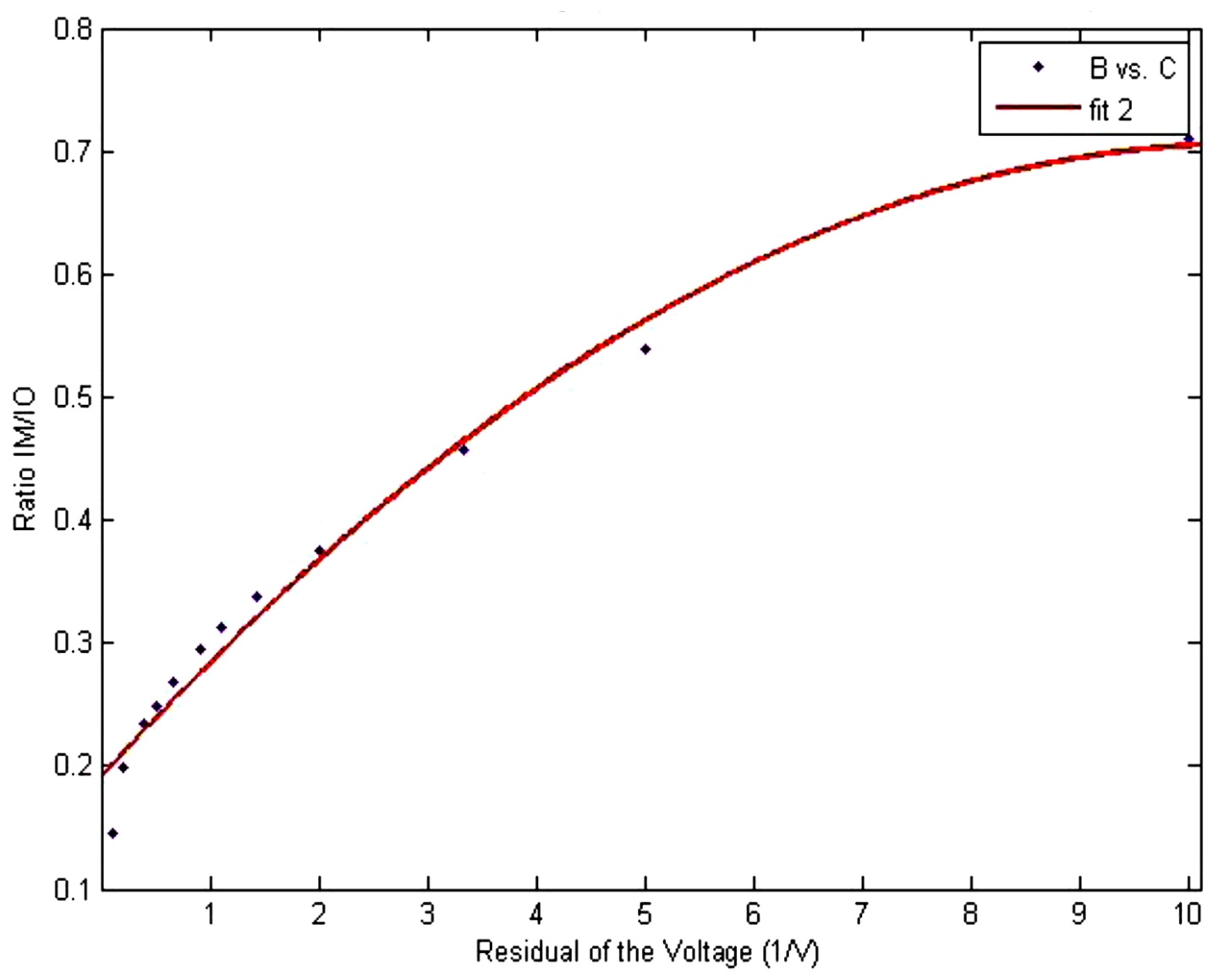

Fig. 5. Current density ratio versus residual of the amplitude of the pulse.

The ratio goes to zero with increasing amplitude. The overshoot current density increases with respect to the stationary current density with the increase amplitude of the voltage pulse indicating that during the time delay of the pulse, plasma has been growing towards the anode. Increasing the time delay, more volume in the anode cathode gap can be filled with the plasma before applying a non-zero voltage to the diode.

This also causes an increment in the initial conductivity of the diode and leads to high current overshoot at the rising end of the output pulse. By increasing the time delay further, the overshoot current will suddenly grow showing a large current with respect to the stationary value i.e. the stationary value can be considered as negligible (Figure 5). At this point the whole A-C gap is filled by the plasma, and the model fails beyond this point.

The value for the delay of the pulse at breaking point of the model depends on the temperature of plasma electrons and their thermal velocity. Therefore by varying the time duration of the pulse only, the plasma boundary follows the curve of erosion which stands for the plasma boundary with an infinite duration pulse.

The limitation of the plasma boundary becomes critical since the plasma boundary can neither exceed the anode-cathode gap nor expand to positive direction of the $\mathrm{x}$-axis, the range following the condition, $0 \leq x \leq d$. By means of the above condition, it is possible to find physically possible ranges for other parameters and these are tabulated in Table 1. 
Table 1. Physically probable limits of the parameters.

\begin{tabular}{|c|c|c|}
\hline Parameter & Range & Other Conditions \\
\hline Amplitude of the pulse $(V)$ & $0 \leq U_{a} \leq 8.1$ & $\begin{array}{c}d=4 \mathrm{~cm}, n_{e}=10^{21} \mathrm{~m}^{-3}, \\
\tau=40 \mathrm{~ns}, U_{d}=10 \mathrm{~ns}\end{array}$ \\
\hline Time delay of the pulse $(\mathrm{ns})$ & $0 \leq \tau \leq 42.5$ & $\begin{array}{c}d=4 \mathrm{~cm}, n_{e}=10^{21} \mathrm{~m}^{-3}, \\
U_{d}=10 \mathrm{~ns}^{2}\end{array}$ \\
\hline Anode Cathode gap $d(\mathrm{~cm})$ & $d \geq 3.75$ & $\begin{array}{c}U_{a}=3 \mathrm{~V}, \mathrm{n}_{e}=10^{21} \mathrm{~m}^{-3}, \\
\tau=40 \mathrm{~ns}, U_{d}=10 \mathrm{~ns}\end{array}$ \\
\hline Electron density $n_{e}\left(\mathrm{~m}^{-3}\right)$ & $0.25 \leq n_{e} \leq 4.23$ & $\begin{array}{c}d=4 \mathrm{~cm}, U_{a}=3 \mathrm{~V}, \\
\tau=40 \mathrm{~ns}, U_{d}=10 \mathrm{~ns}\end{array}$ \\
\hline Electron Thermal Energy $(\mathrm{eV})$ & $0.1 \leq T_{e} \leq 5.6$ & $\begin{array}{c}d=4 \mathrm{~cm}, n_{e}=10^{21} \mathrm{~m}^{-3}, \\
\tau=\mathrm{ns}, U_{d}=10 \mathrm{~ns}, \mathrm{U}_{a}=3 \mathrm{~V}\end{array}$ \\
\hline
\end{tabular}

\section{SIMULATION BY DIFFERENT PERIODIC VOLATGE SIGNALS}

The modeled plasma diode was simulated with sine, square, triangle and saw tooth periodic voltage functions. The equations generating these periodic functions are given in the Table 2. The periodic patterns which can be generated by the model are shown in the Figure 6 . The input voltage pattern was changed by keeping the amplitude at $4000 \mathrm{~V}$, frequency at $10^{8} \mathrm{~Hz}$ and A-C gap at $4 \mathrm{~cm}$, electron number density at $10^{17} \mathrm{~m}^{-3}$ and temperature at $4000 \mathrm{~K}$.

Table 2. Fourier series of wave patterns.

\begin{tabular}{|c|c|}
\hline Wave Pattern & Fourier Series \\
\hline Square wave & $\frac{4}{\pi} \sum_{n=0}^{\infty} \frac{\sin (2 n+1) x}{(2 n+1)}$ \\
\hline Absolute sine wave & $\frac{2}{\pi}-\frac{4}{\pi} \sum_{n=1}^{\infty} \frac{\cos 2 n x}{4 n^{2}-1}$ \\
\hline Triangular wave & $\frac{8}{\pi^{2}} \sum_{n=0}^{\infty} \frac{\cos (2 n+1) x}{(2 n+1)^{2}}$ \\
\hline Rectangular saw-tooth wave & $\frac{L}{\pi} \sum_{n=1}^{\infty}(-1)^{n-1} \frac{\sin n x}{n}$ \\
\hline
\end{tabular}




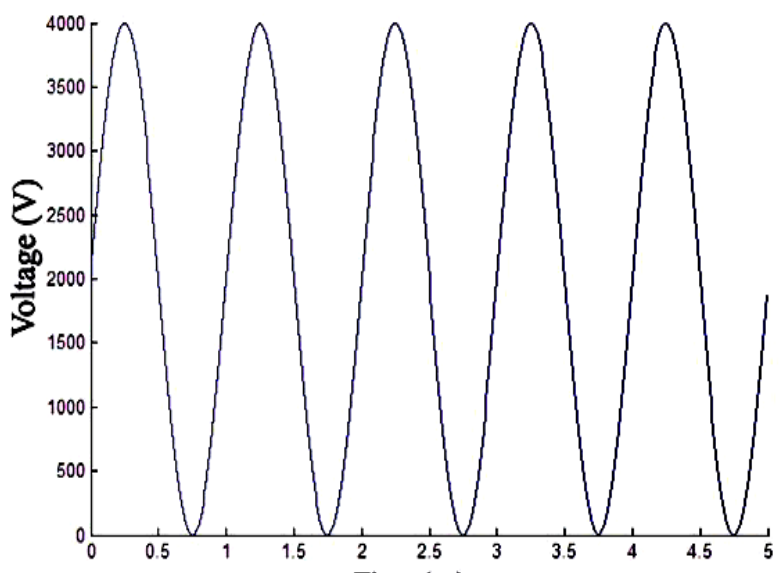

(a)

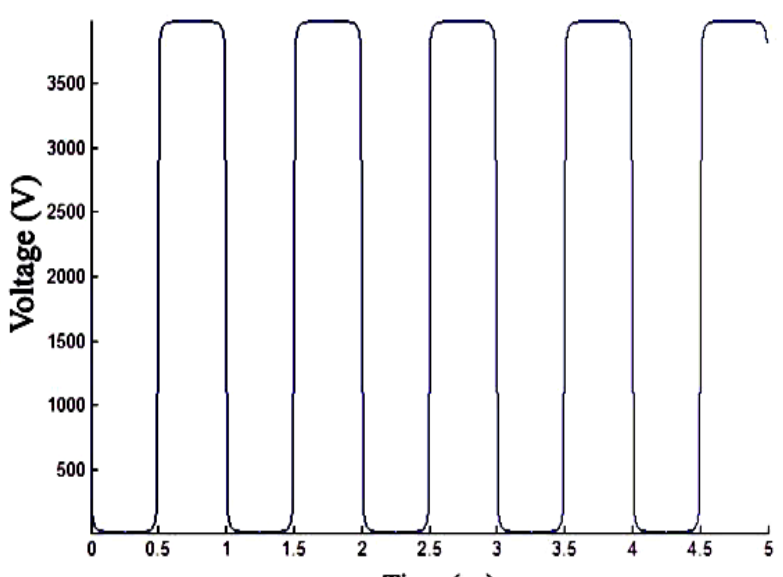

(b) Time (ns)

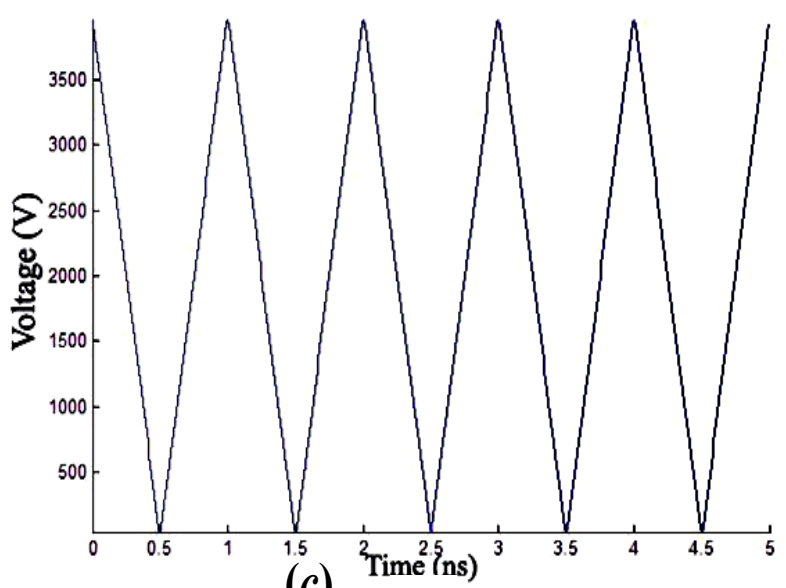

(c)

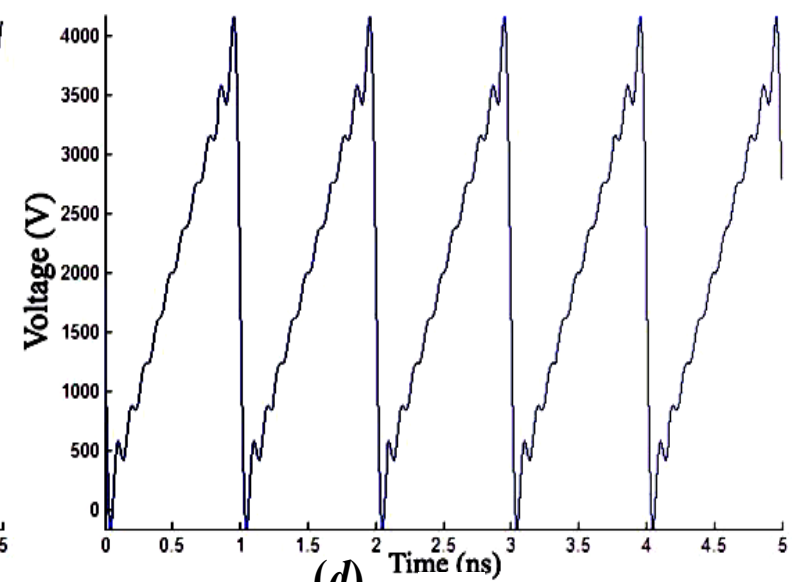

(d)

Fig. 6. Input voltage pattern: $(a)$ sine function, $(b)$ square function, $(c)$ triangular function and $(d)$ saw-tooth function.

The input voltage patterns and the plasma boundary are shown in the Figures 7 . For all voltage patterns, the movements of the plasma boundary determined by the plasma and electron current density showed that the plasma boundary is moving towards the anode.

The plasma is expanding when the applied voltage is less than the equilibrium voltage, the sufficient voltage that keeps the plasma boundary. The plasma boundary move back towards the cathode (plasma erosion) when the applied voltage is greater than the equilibrium voltage. In each case, the overall movement of the plasma boundary leads to an plasma expansion. But as the average poistion of the plasma boundary was moving towards the anode, the equilibrium level was reduced and the average movement of plasma boundary oscillates around a stationary poition.

Since the average position of the plasma boundary has move towards the anode, the conductivity of the diode increased, thereby the peaks current also increased with time and reached a stationary value. Since sine and the triangular voltage patterns have nearly similar forms, the behaviour of plasma boundaries was similar. 


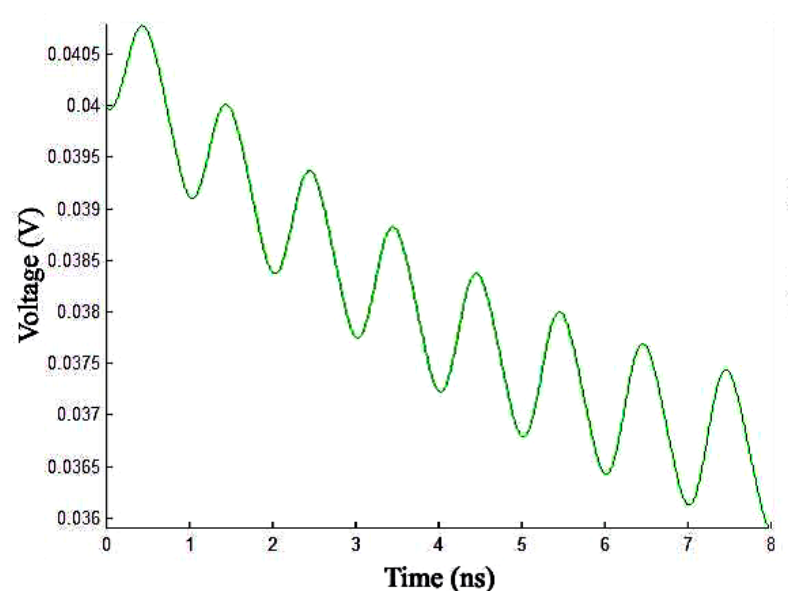

(a)

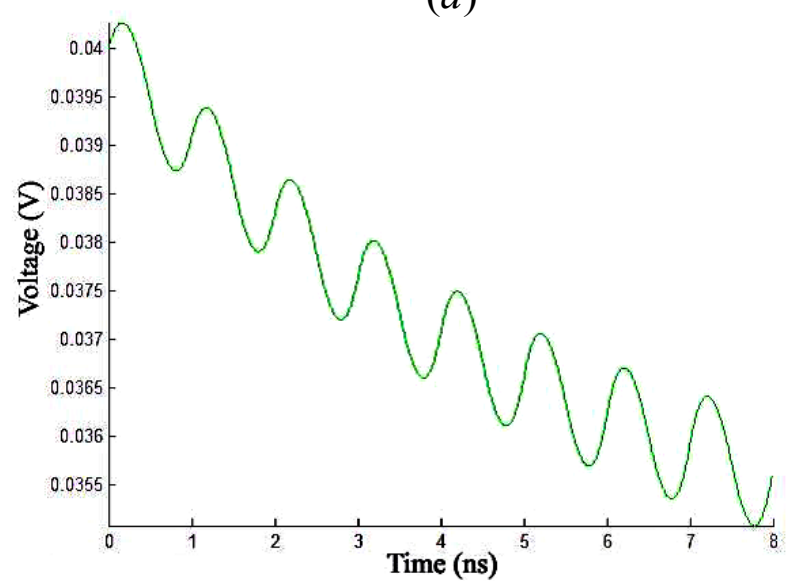

(c)

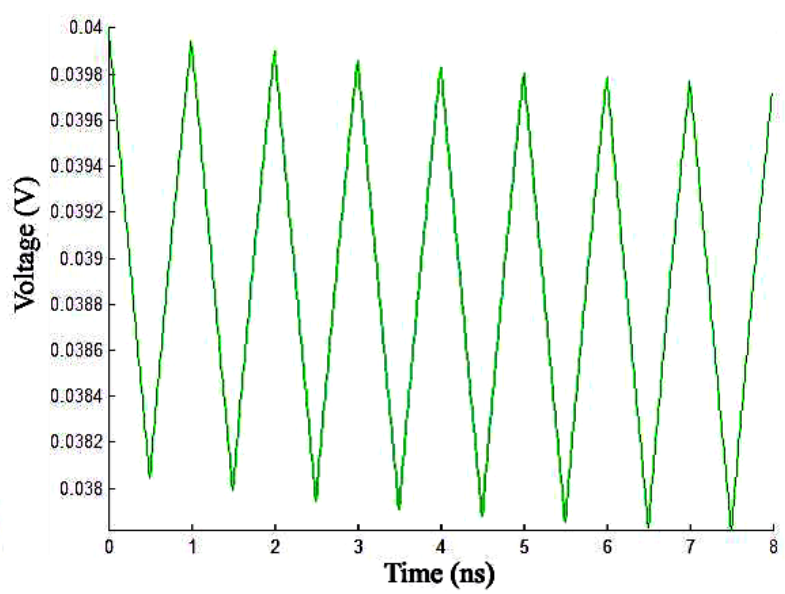

(b)

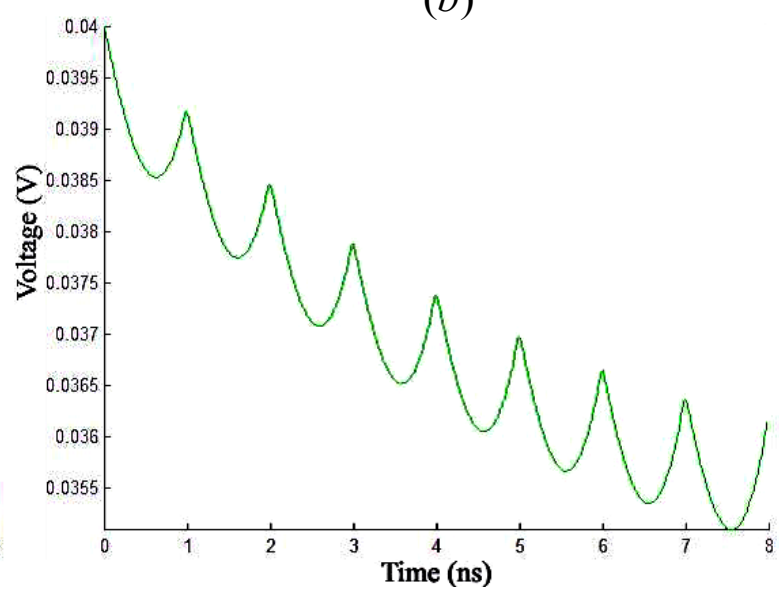

(d)

Fig. 7. Behaviour of the plasma boundary with different voltage patterns: $(a)$ sine function, $(b)$ square function, $(c)$ triangular function and $(d)$ sawtooth function.

In the case of square voltage pattern which have only two voltage levels, the plasma expand with constant velocity during the period of zero voltage and erode with constant velocity during the period of non-zero voltage giving rise to a triangular behavior of the plasma boundary.

Whereas for the sawtooth voltage, as the input voltage is changing rapidly the variation of the plasma boundary is not smooth.

By changing the input voltage amplitude but keeping the other parameters constant, the current densities through the diode and the plasma boundary for the voltage patterns are presented in Figure 8.

The peak value and the average boundary value can be changed by varying the amplitude of the pulse. 

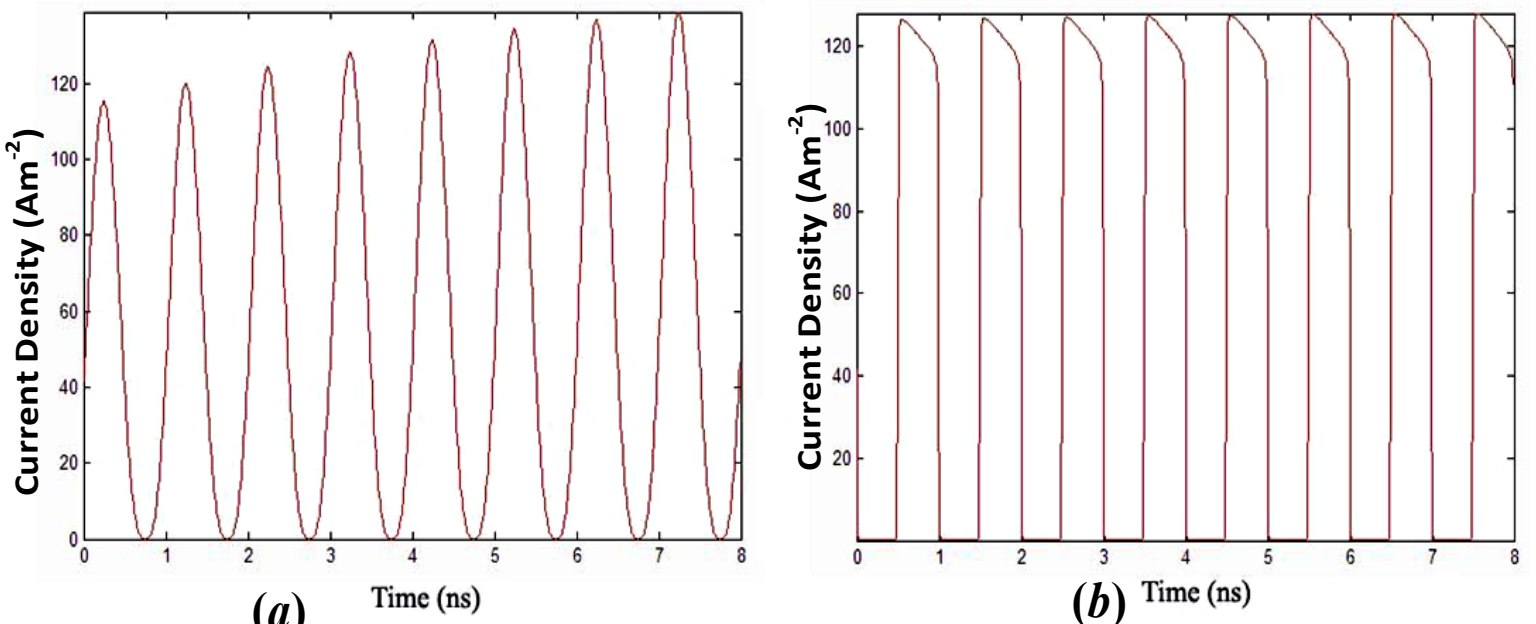

(b) Time (ns)

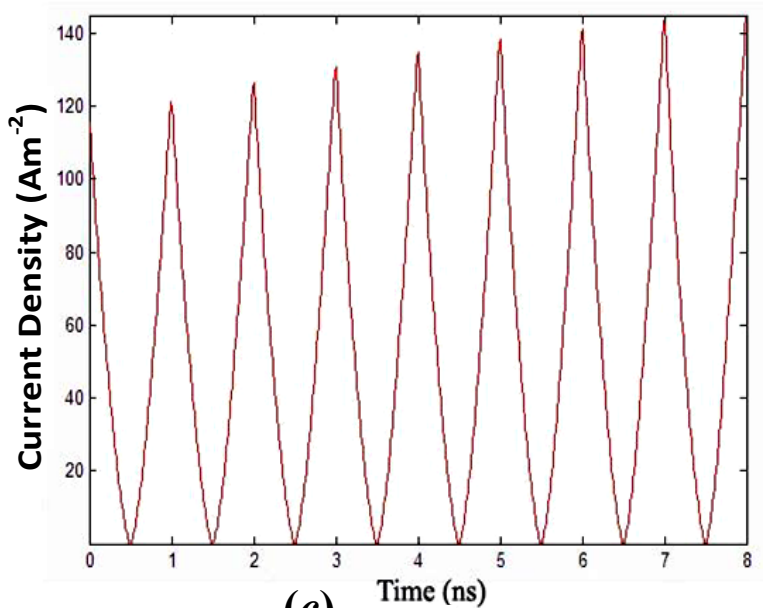

(c)

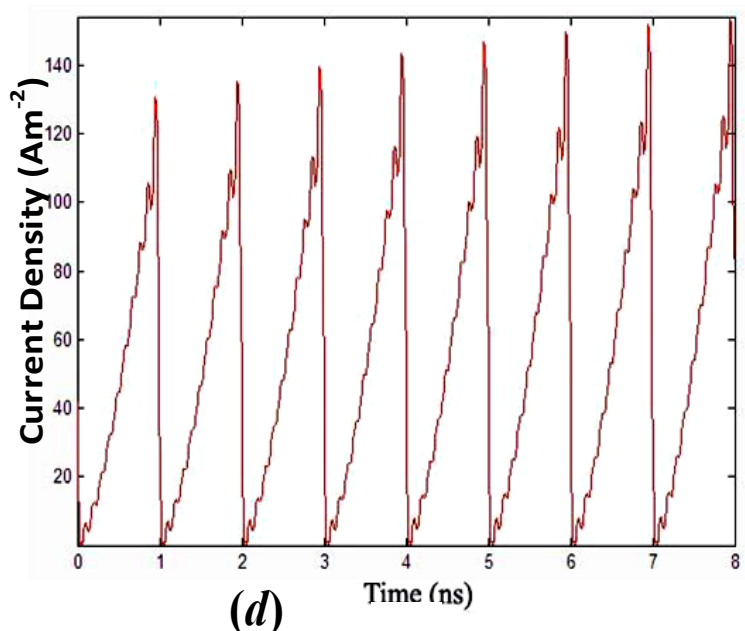

Fig. 8. Behaviour of the current density with different voltage patterns: $(a)$ sine function, $(b)$ square function, $(c)$ triangular function and $(d)$ sawtooth function.

The voltage amplitudes at stationary points for sine, square triangular and sawtooth pattern are at 4800, 4050, 4850 and $4750 \mathrm{~V}$ respectively. The graphs obtained for the best curve fitting models of the plasma boundary for different input sine voltage amplitudes in the range of $2000 \mathrm{~V}$ to $10,000 \mathrm{~V}$ is shown in Figure 9.

The average stationary plasma boundary with time for sine waves is between the voltage amplitude range $4700 \mathrm{~V}$ to $5000 \mathrm{~V}$. For amplitudes above the stationary point, the plasma had expanded in opposite direction of cathode to anode and has an upper bounded value.

Plasma boundary and current density with different frequencies were investigated for three different frequency sine voltage patterns. The curve fitting models were found for these and are illustrated in figure 10. A significant relationship between the plasma boundary and the frequency of the input voltage pattern is not apparent. 


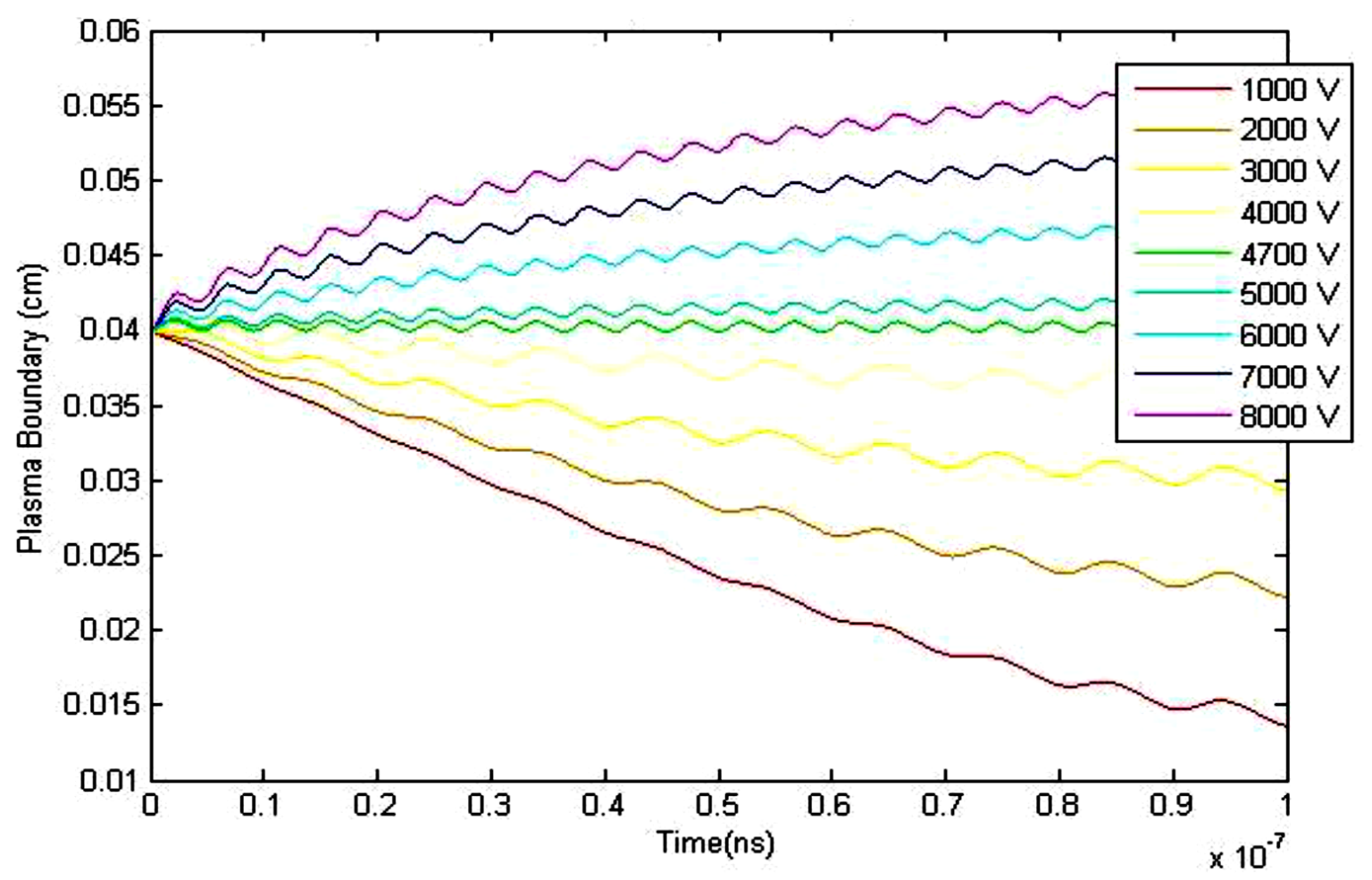

Fig. 9. Average plasma boundary vs. time for different input sine voltages.

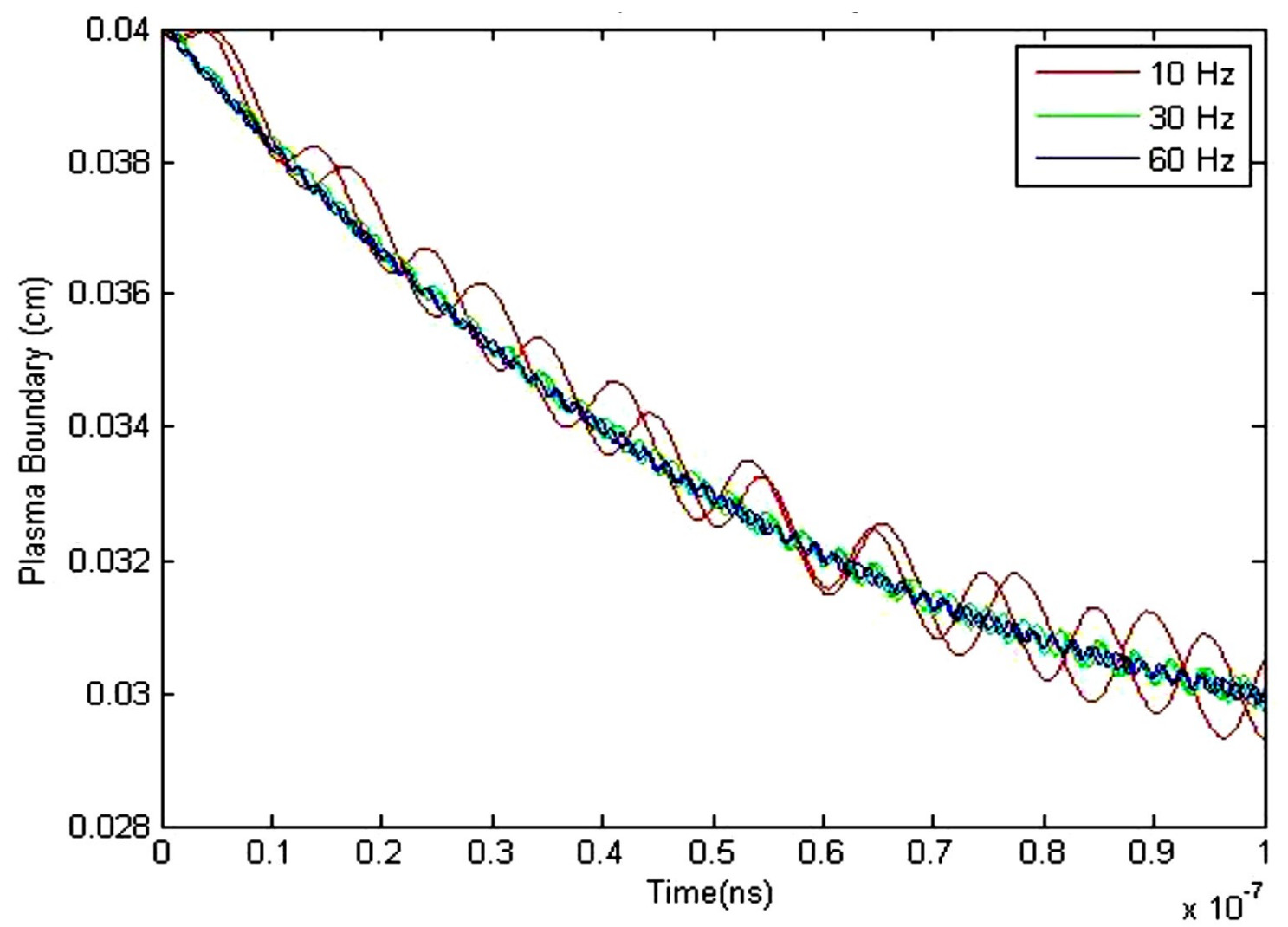

Fig. 10. Plasma boundary vs. time for different frequencies of sine voltages. 
The electron densities at the stationary point or lower bound of the electron density was measured by varying sine voltage amplitude of the pulse in a range $2000 \mathrm{~V}-10000 \mathrm{~V}$ and A$\mathrm{C}$ gap in $2.2 \mathrm{~cm}-4.7 \mathrm{~cm}$. The lower bound value of the electron number density over amplitude of the pulse and the A-C gap are depicted in the Figure 11.

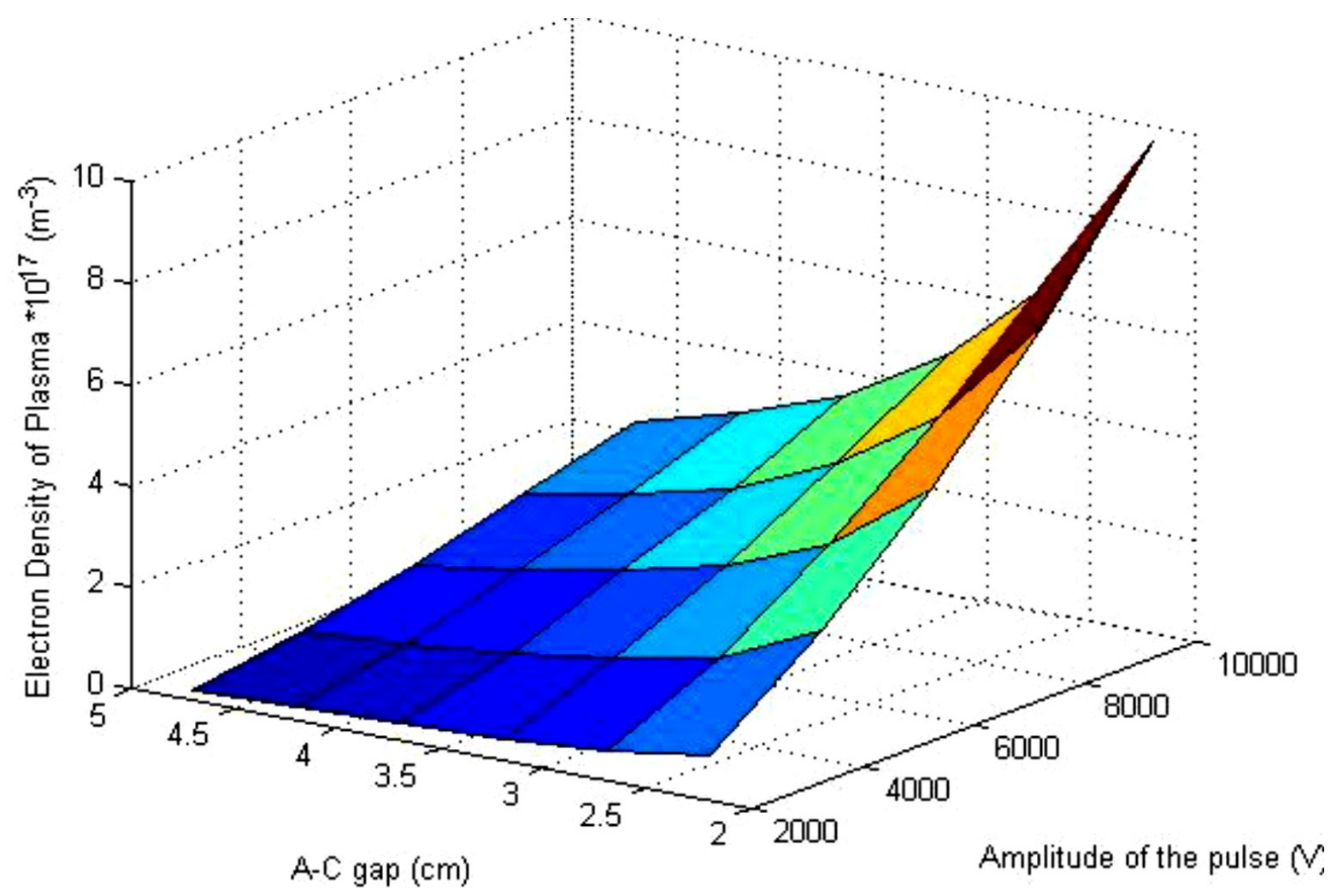

Fig. 11. Current densities for different input sine voltage amplitudes and AC gaps.

The data was analyzed by using a two dimensional interpolation technique and a new set of interpolated data is shown in Figure 12. By means of the above generated data, it was possible to see the regression of minimal electron density of the system with amplitude of the pulse and Ac gap.

The interpolated data illustrated in a contour plot in Figure 13, can be used to find lower bound of the electron density of the plasma which is generated inside the anode cathode gap for specified plasma diode system.

Thus if the maximum amplitude of the application voltage and the anode cathode gap are known, the minimal electron density of a plasma can be estimated.

The current densities over time with different amplitudes of applied sine voltage pulse are presented in Figure 14. 


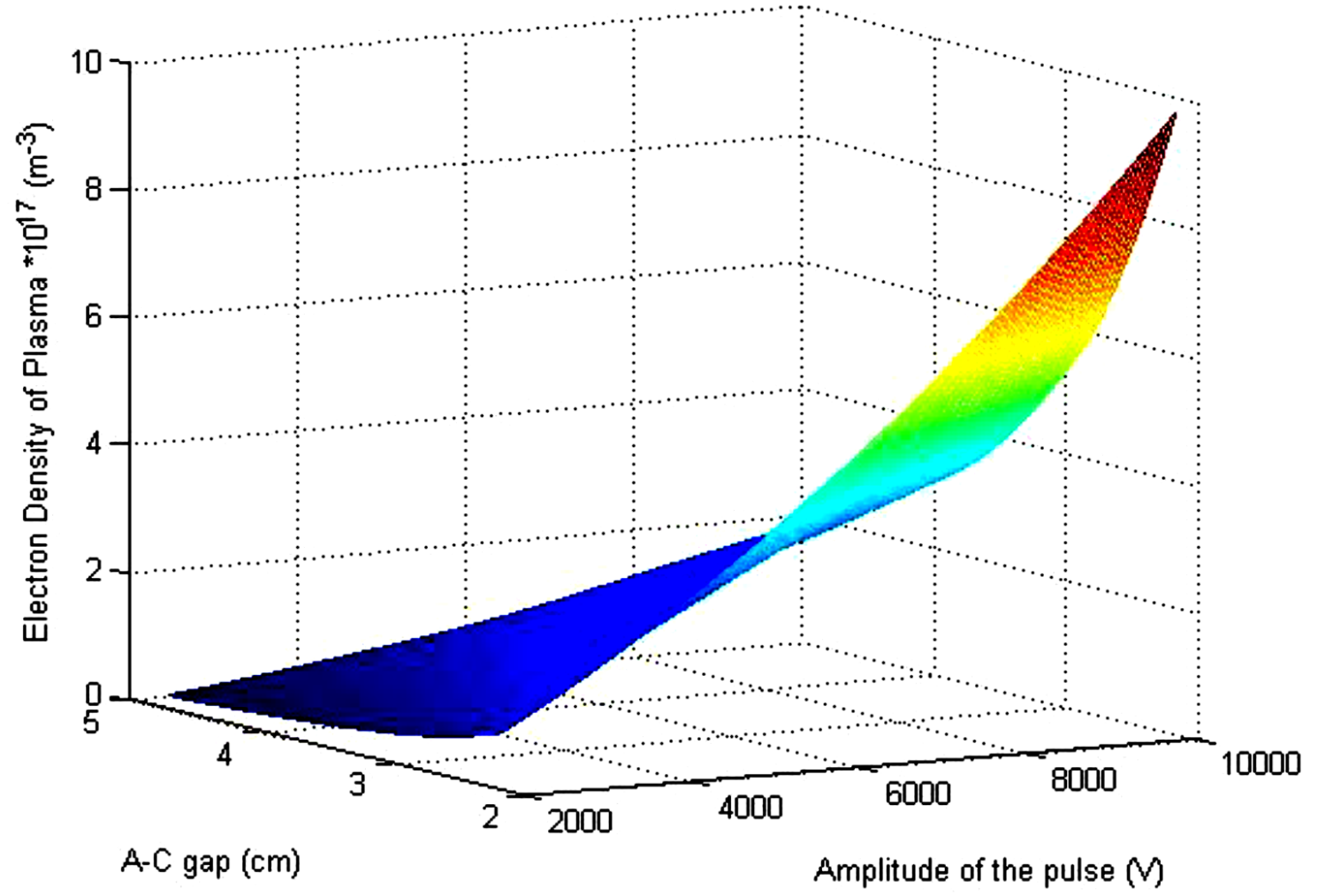

Fig. 12. Three dimensional plot of interpolated data.

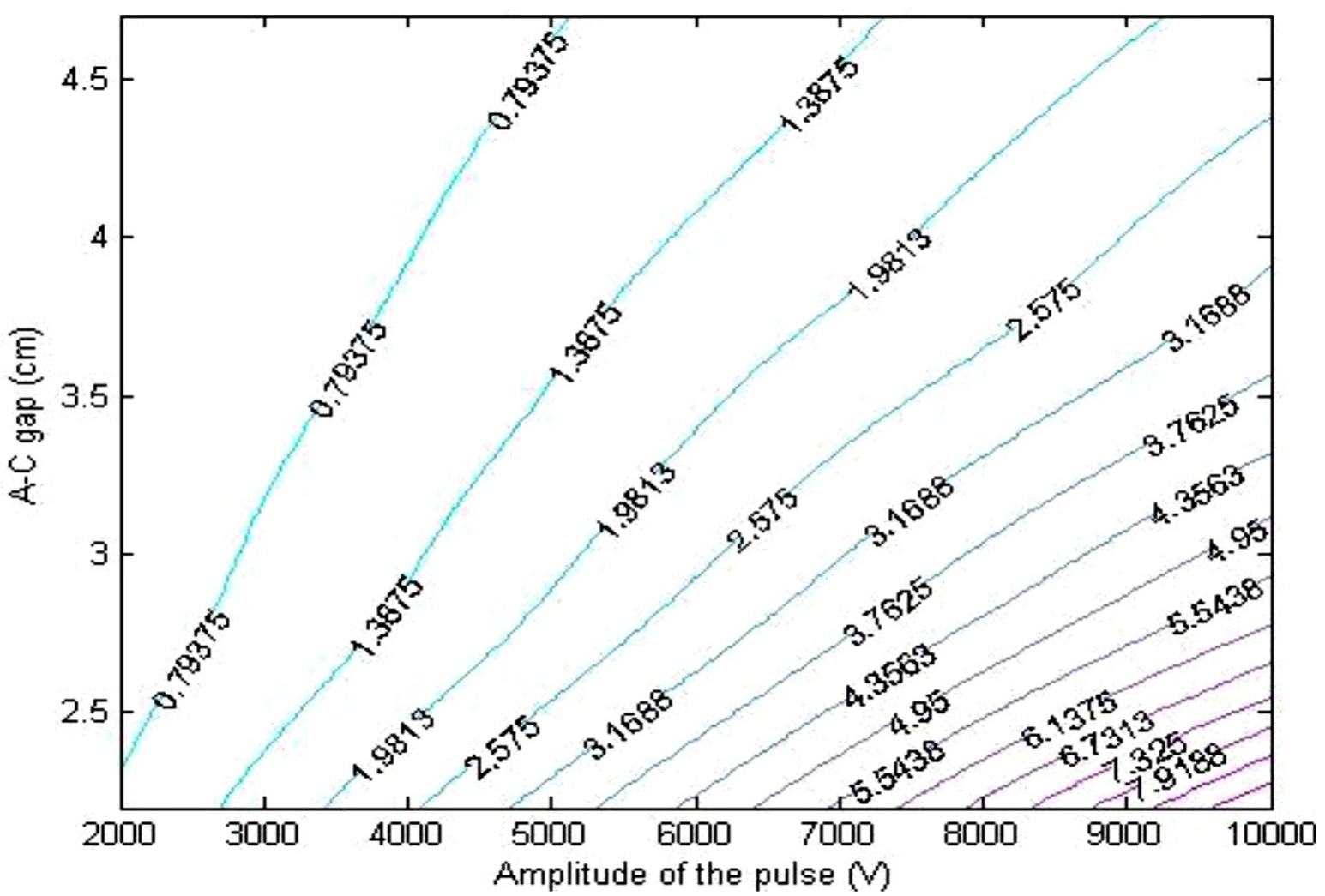

Fig. 13. Contour plot of electron number density with amplitude of the pulse and the A-C gap of the diode. 


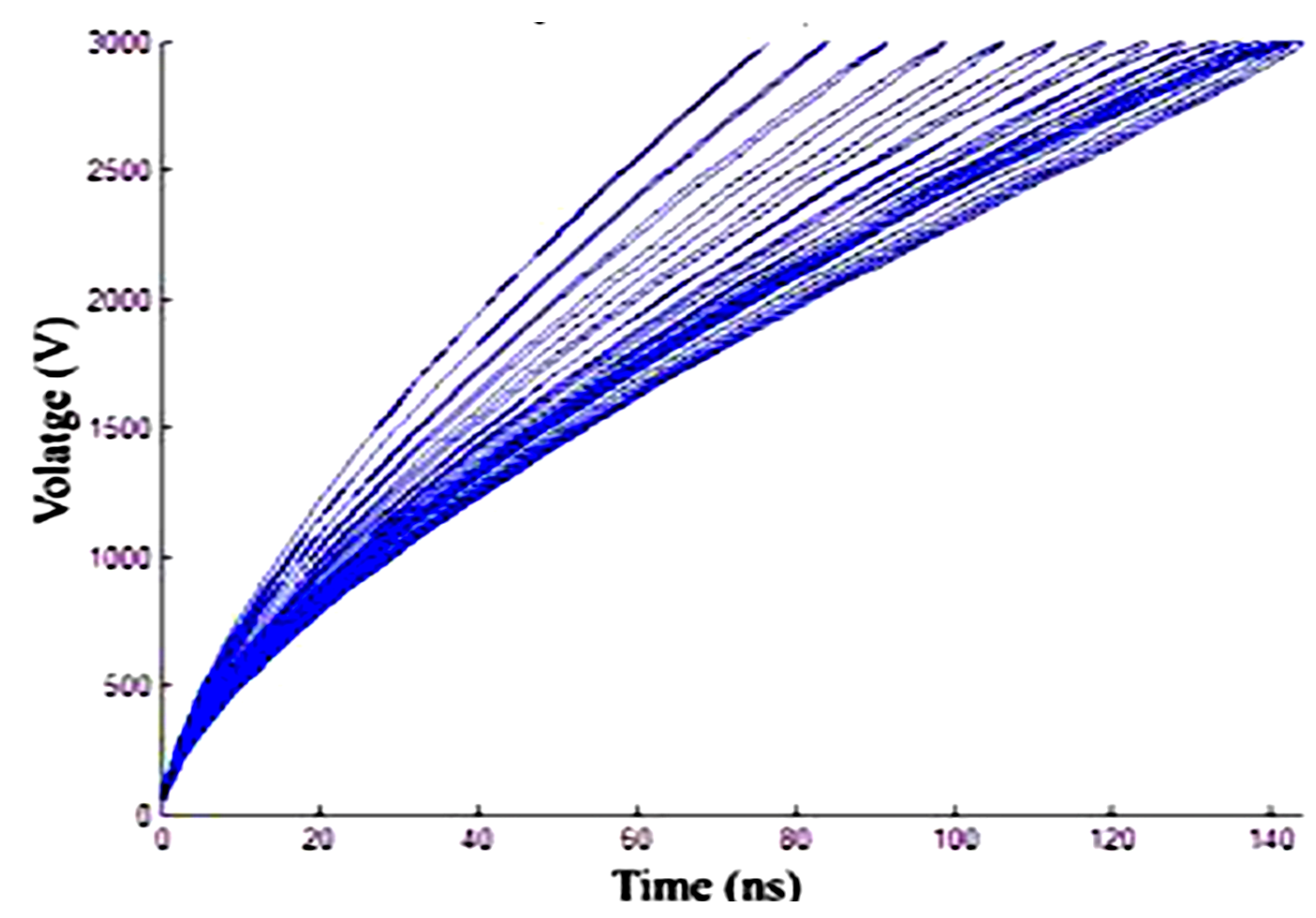

(a) Amplitude 3000V

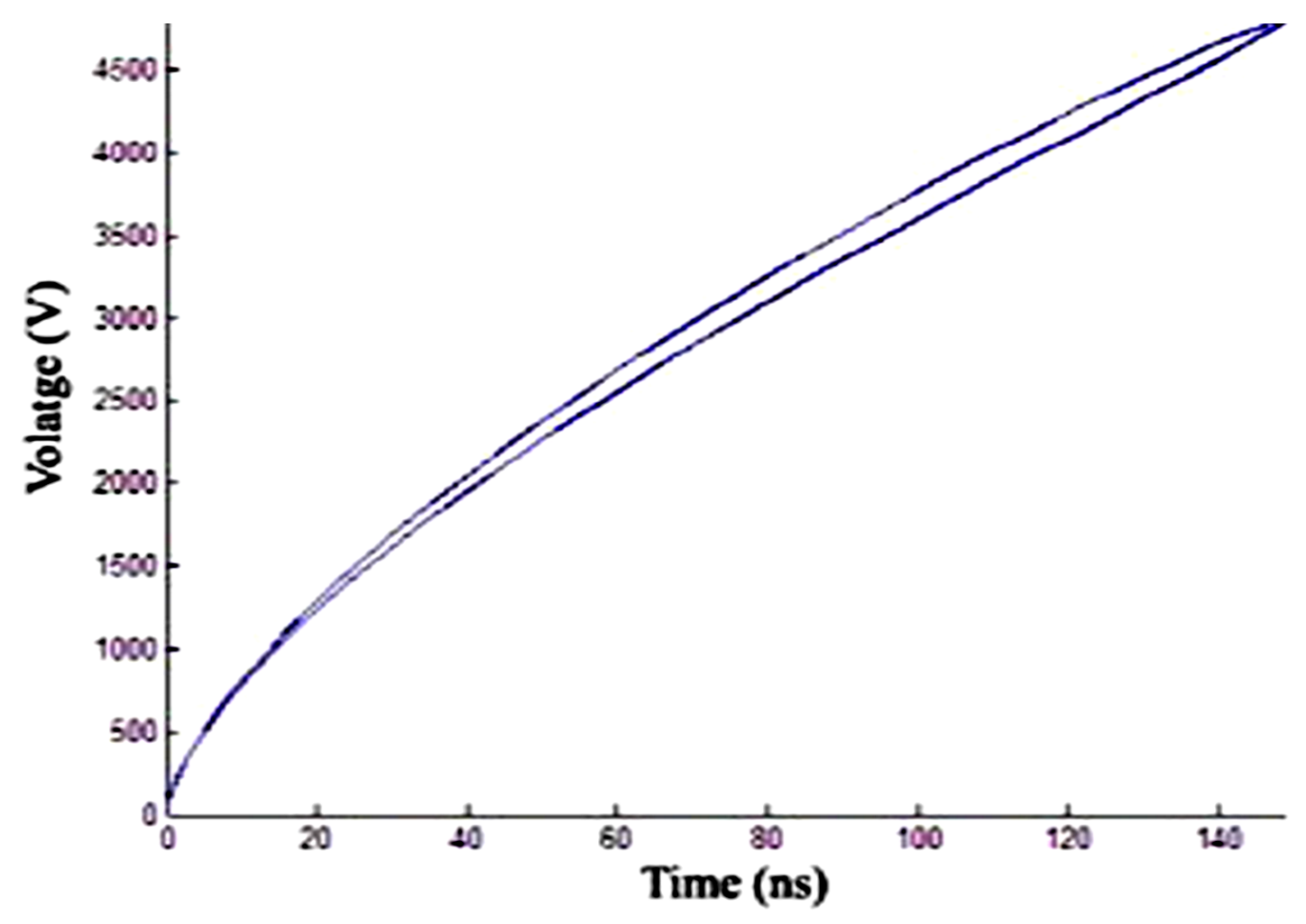

(b) Amplitude 4800V 


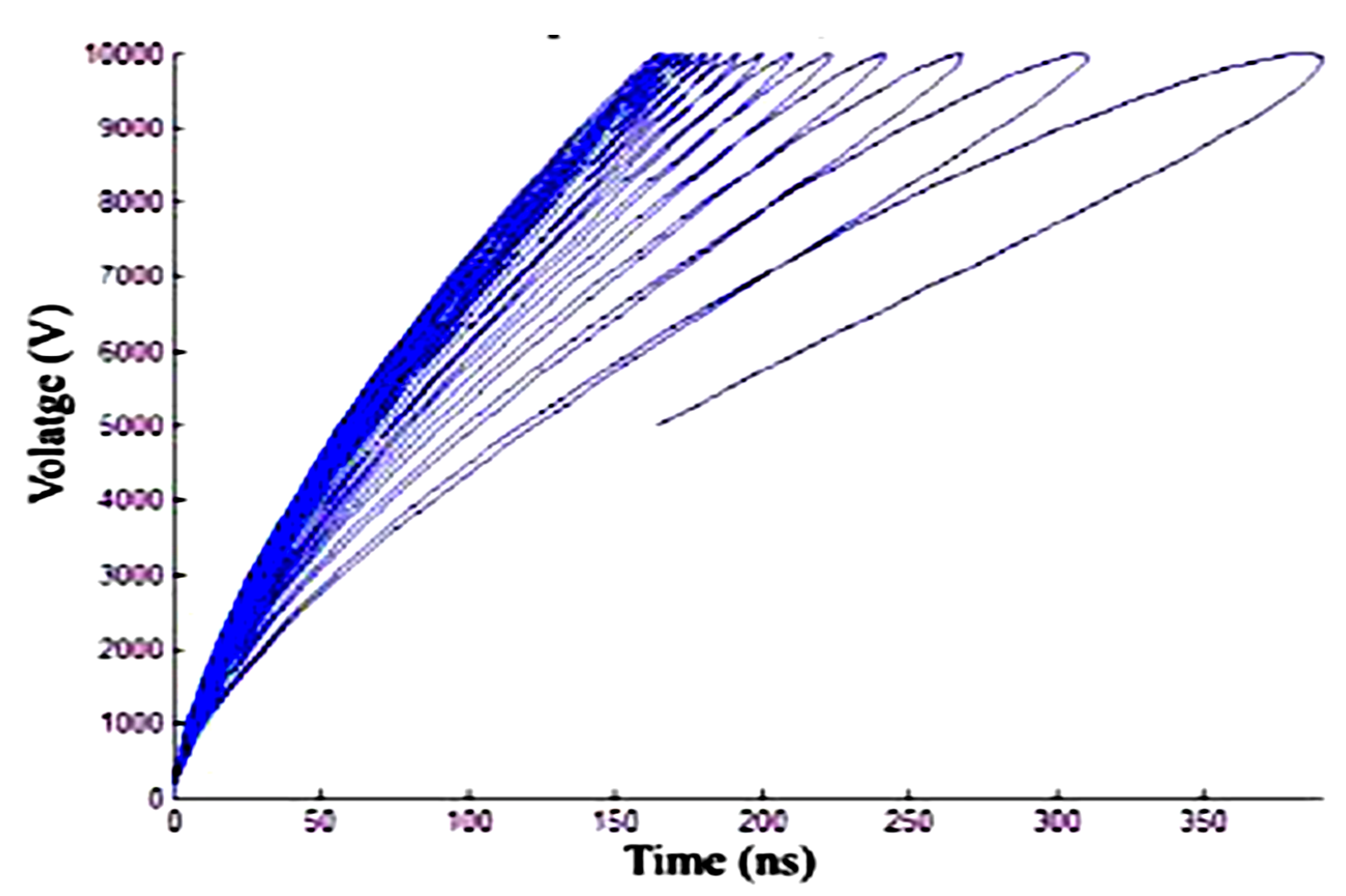

(c) Amplitude 10,000V

Fig. 14. Current densities for different input sine voltage amplitudes of $(a) 3000 \mathrm{~V}$, (b) $4800 \mathrm{~V}$ and (c) $10,000 \mathrm{~V}$.

These feather like patterns fold or untwist with the increasing or decreasing amplitudes. Although the pattern obtained for all the four types of voltage patterns were similar each type has its own shape for the branches of the feather. The amplitude of the pulse was increased from the lowest possible value that the model supports by keeping the other parameters of the system as constants. The feather like pattern folded first from an untwisted pattern and all its braches collapsed together to a single branch at the stationary point of the system. With the further increment of the amplitude, the feather like pattern began to open up from the single branch. The heads of the branches reduce gradually in anti-clock wise direction. For amplitudes less than stationary point, more branches are concentrated around lower voltage region of feathers i.e. right end of the feathers while for amplitudes greater than stationary point more branches are concentrated around higher voltage region, i.e. left end of the feathers.

\section{PARTICLE IN CELL SIMULATION}

A particle-in-cell technique [10] was used to model the plasma inside the plasma diode. The time evolution of finite sized macro particles also known as computational particles was predicted by a self-consistent calculation of the field equation defined for each of the macro particle $i$ of the system by, 


$$
\frac{\partial v_{i}}{\partial t}=\frac{\partial x_{i}}{\partial t^{2}}=\left(\frac{e}{m_{e}}\right) \frac{\partial \phi}{\partial x}
$$

Particle-in-cell utilizes a set of computational particles to represent the transport of locally conserved quantities like charge and mass. The dynamics of a system of charge particles is given by the Vlasov equation for the one particle distribution function,

$$
\frac{\partial f_{s}}{\partial t}+v \frac{\partial f_{s}}{\partial x}+\frac{q E}{m} \frac{\partial f_{s}}{\partial v}=0
$$

where $f_{s}$ is the phase space distribution function for a given species $s$ (ion or electron) which represents the number density per unit element of the phase space or the probability of finding a particle in the area $d x \times d v$ around a certain phase space point $(x, v)$. But the Vlasov equation does not stands for the collision effect of the particles in the system [11]. The charge density distribution function is given by

$$
\rho(x, t)=\sum_{s} f_{s}(x, v, t) d v .
$$

The mathematical formulation of the particle-in-cell method is obtained by assuming that the distribution function of each species is given by the superposition of several elements named as computational particles. Each element represents a large number of physical elements that are near each other in phase space.

$$
f_{s}(x, v, t)=\sum_{p} f_{p}(x, v, t)
$$

where $f_{s}$ is the phase space distribution function and $f_{p}$ is the distribution function of physical particles. This method is based upon assigning to each computational particle, a specific functional form for its distribution and with a number of free parameters whose time evolution will determine the numerical solution of the Vlasov equation. The choice is usually made to have two free parameters in the functional shape for each spatial dimension acquiring the physical meaning of position and velocity of the computational particle. The functional dependence is further assumed to be the tensor product of the shape in each direction of the phase space,

$$
f_{p}(x, v, t)=N_{p} S_{x}\left(x-x_{p}(t)\right) S_{v}\left(v-v_{p}(t)\right)
$$

where $S_{x}$ and $S_{v}$ are the shape functions for the computational particles and $N_{p}$ is the number of physical particles that are present in the element of phase space represented by computational particle. The spatial shape functions are based on the use of $b$-spline functions, a series of consecutively higher order functions obtained from each other by integration. The 
subsequent $b$-splines $\left(b_{l}\right)$, can be obtained by successive integration of the following generating formula,

$$
b_{l}=\int_{-\infty}^{+\infty} b_{0}\left(\xi-\xi^{\prime}\right) b_{l-1}\left(\xi^{\prime}\right) d \xi^{\prime}, \quad b_{0}(\xi)=\left\{\begin{array}{c}
1 \text { if }|\xi|<1 / 2 \\
0 \text { otherwise }
\end{array}\right.
$$

By means of the $b$-splines, the spatial shape function is chosen as:

$$
S_{x}\left(x-x_{p}\right)=b_{l}\left(\frac{x-x_{p}}{\Delta p}\right)
$$

where $\Delta p$ is the size of the computational particles. Equations of motion for the computational particles are given by

$$
\frac{d N_{p}}{d t}=0, \quad \frac{d^{2} x_{p}}{d t^{2}}=\frac{d v_{p}}{d t}=\frac{q_{s}}{m_{s}} E_{p} .
$$

The numerical solution of Poisson and Vlasov equation was obtained using finite difference method assuming a grid of equal cells size $\Delta x$ with cell centres $x_{i}$ and cell vertices $x_{i+1 / 2}$ (Figure 15).

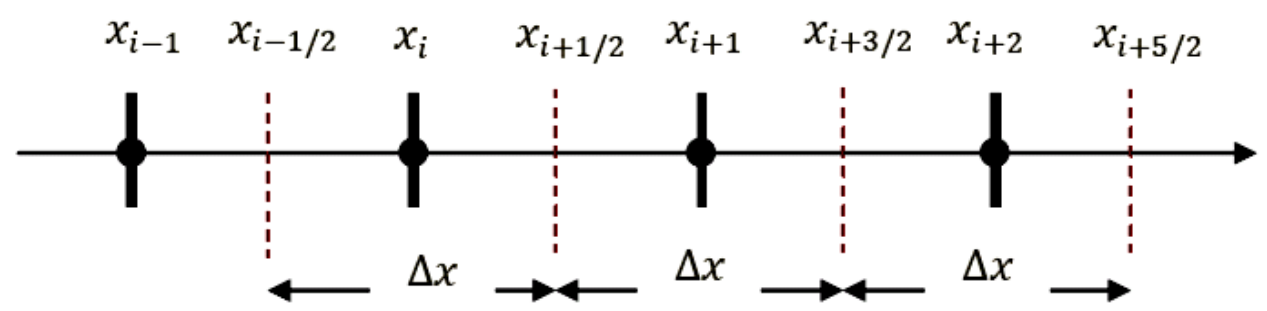

Figure 15. 1D grid of equal cells.

The scalar potential $\phi$ is introduced by the cell averaged values $\phi_{i}$. Poisson equation can be solved by means of three point formula,

$$
\varepsilon_{0} \frac{\phi_{i+1}-2 \phi_{i}+\phi_{i-1}}{\Delta x^{2}}=\rho_{i}
$$

where the densities $\rho_{i}$ are similarly defined as average over the cells $\left[x_{i-1 / 2}, x_{i+1 / 2}\right]$. The electric field was computed in cell canters by using the discrete potentials and the continuum electric field can be reconstructed using the assumption that the field is constant in each cell and equal to its average value, 
$E(x)=\sum_{i} E_{i} b_{0}\left(\frac{x-x_{i}}{\Delta x}\right), \quad E_{i}=-\frac{\phi_{i+1}-\phi_{i}}{\Delta x}$

The simplest algorithm used to solve the equations of motion is based on staggering the time level of velocity and position by half time step, the leap-frog algorithm. The advancement of the position from time level $n$ to $n+1$ uses the velocity at mid point $v_{p}^{n+1 / 2}$ and similarly, the advancement of the velocity from time level $n-1 / 2$ to $n+1 / 2$ uses the midpoint position $x_{p}^{n}$ as show in the Figure 16 .

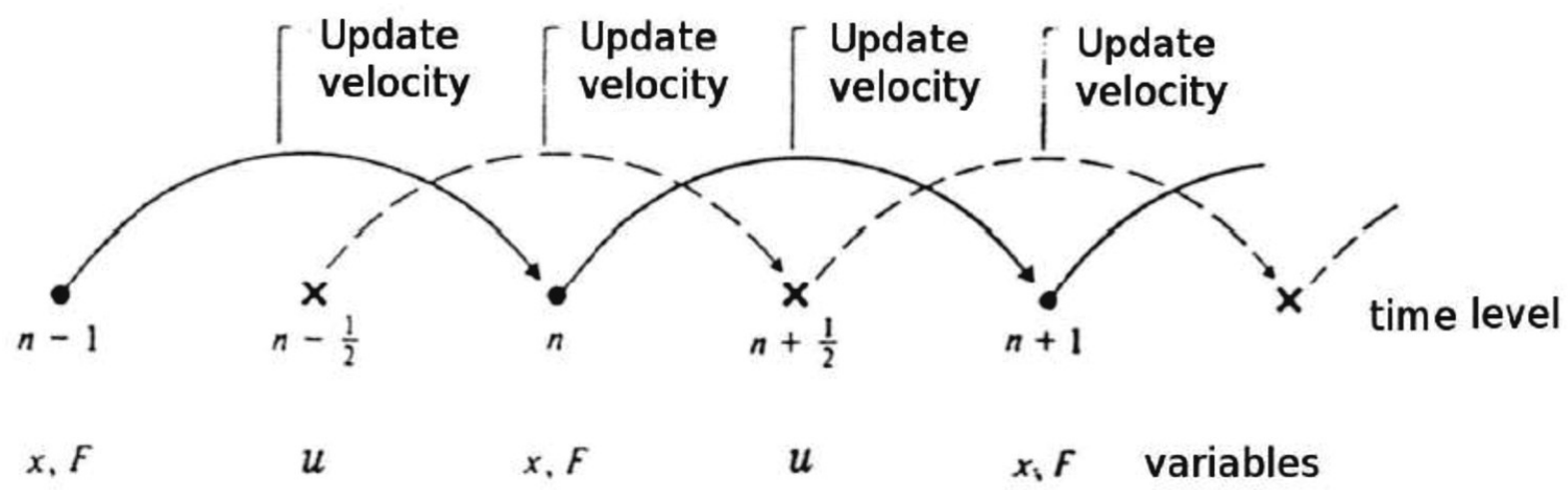

Figure 16. Visual representation of the leap-frog algorithm.

Therefore

$x_{p}^{n+1}=x_{p}^{n}+\Delta t v_{p}^{n+1 / 2}, \quad v_{p}^{n+3 / 2}=v_{p}^{n+1 / 2}+\Delta t E_{p}\left(x_{p}^{n}\right)$

The charge distribution over the A-C length was calculated by assuming that the total cell charge of the grid was uniformly distributed over the cell area around the centralized computational particle at the middle of the cell so that the particles were located uniformly in random positions. The velocities of the particles were calculated using the Gaussian normal distribution function with a standard deviation of 0.5 for random particles. The charge density $\rho(x)$ and potential $\phi(x)$ are periodic and from the relation between their discrete Fourier transformations given by Poisson equation $\phi(k)=\rho(k) / \varepsilon_{0} k^{2}$, the effective electric field on each computational particle was found by using fast Fourier and inverse fast Fourier transformation techniques. The electric field data was interpolated by means of cubic spline interpolation method to find the Lorentz's forces acting on particles and acceleration and new velocity and new position of each particle were calculated. Simulation was done for a plasma of 2000 electrons and 500 hydrogen, for a time period of $0.2 s$ with 1000 iterations.

Although the particle collisions with the boundaries (anode or cathode) were investigated during the simulation period, the inter-particle collisions were ignored due to their complexity. After each $0.2 \mathrm{~ms}$ time step, electric field distribution without and with an 
external electric field of $E_{\text {ext }}=0.5 \times 10^{-25} \mathrm{Vm}^{-1}$ is shown in Figures 17 and the particles in a phase space diagram in Figure 18.

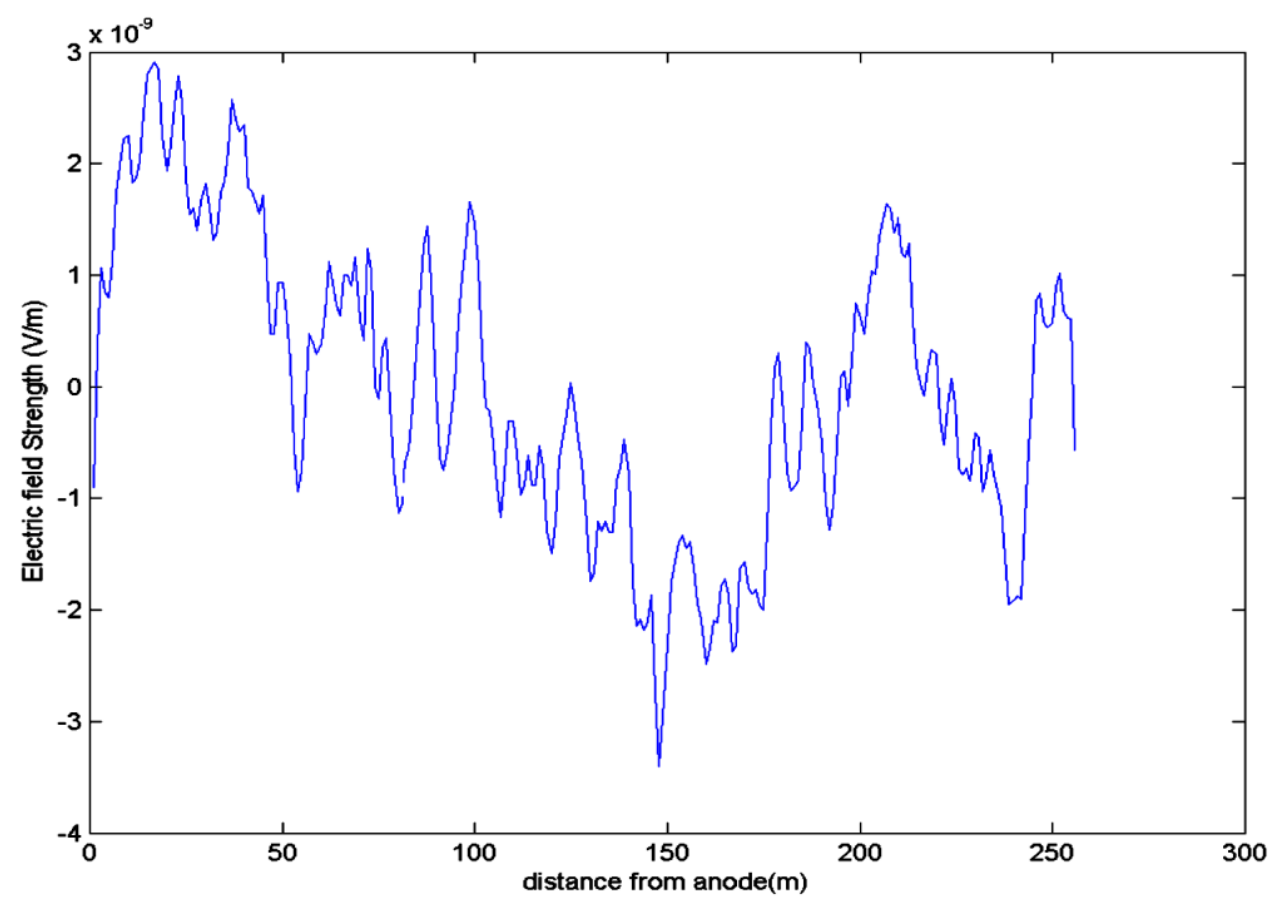

(a) $t=0.019 s, \quad E_{\text {ext }}=0$

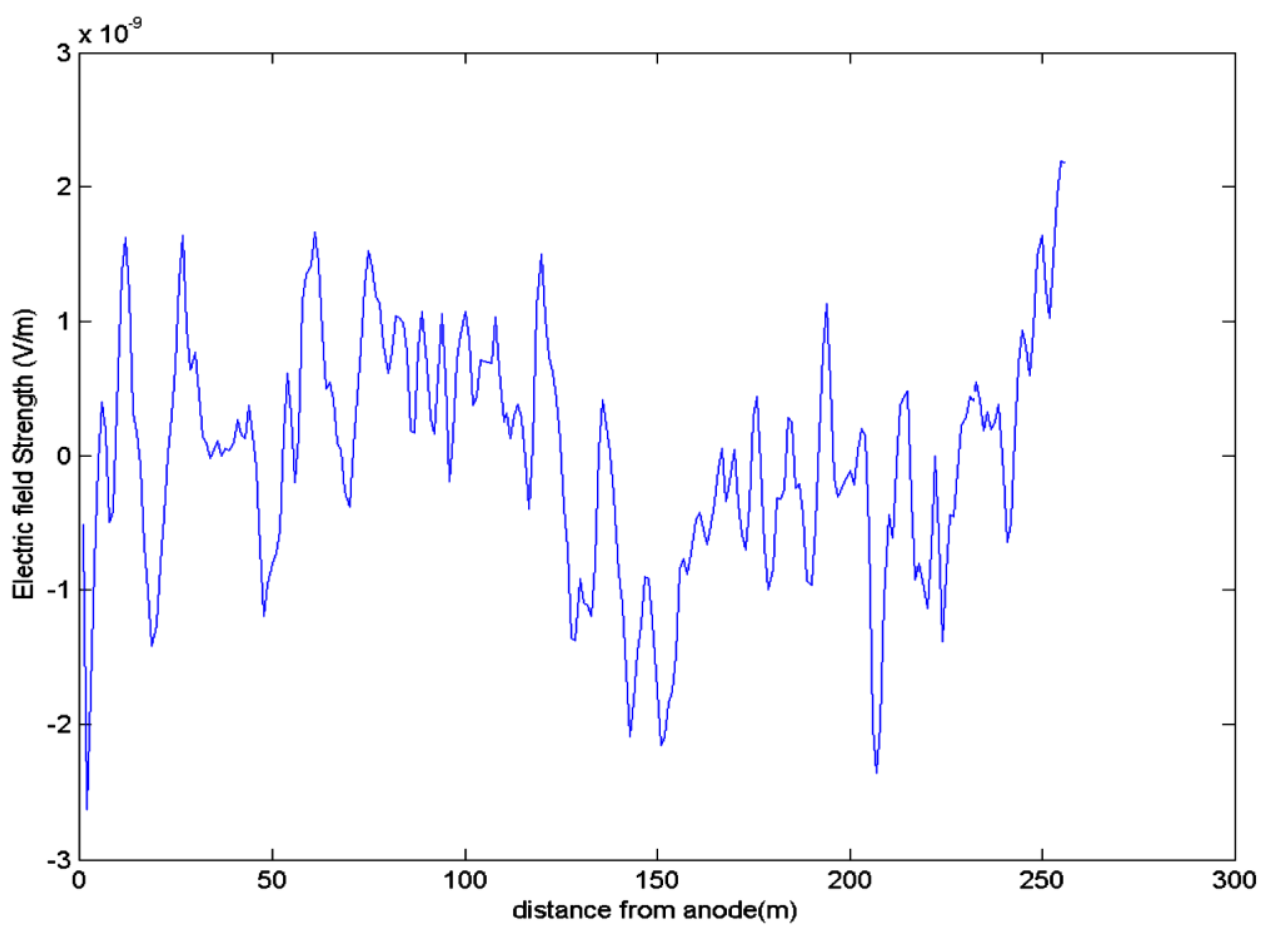

(b) $t=0.199 s, \quad E_{\text {ext }}=0$ 


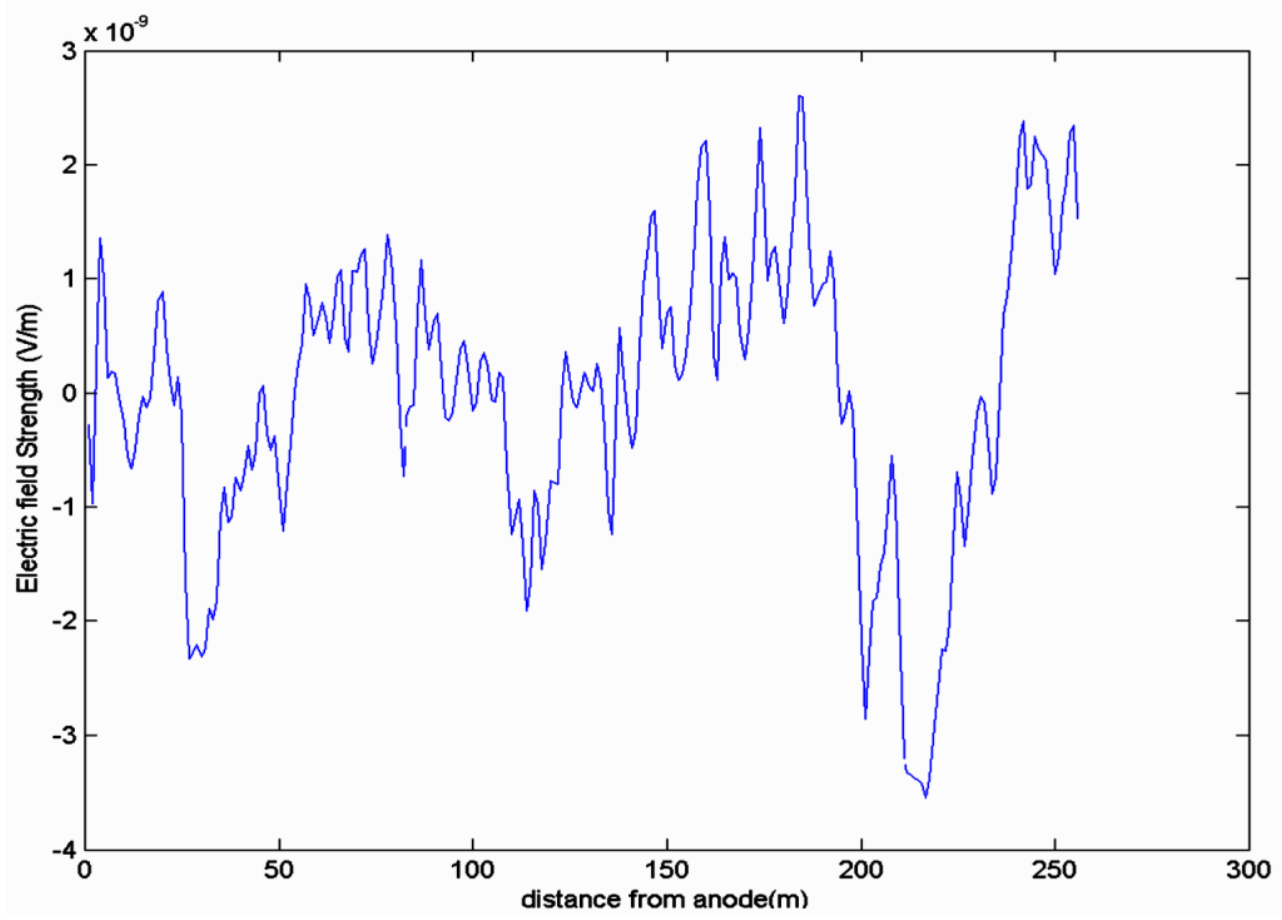

(a) $t=0.019 \mathrm{~s}, E_{\text {ext }}=0.5 \times 10^{-25} \mathrm{Vm}^{-1}$

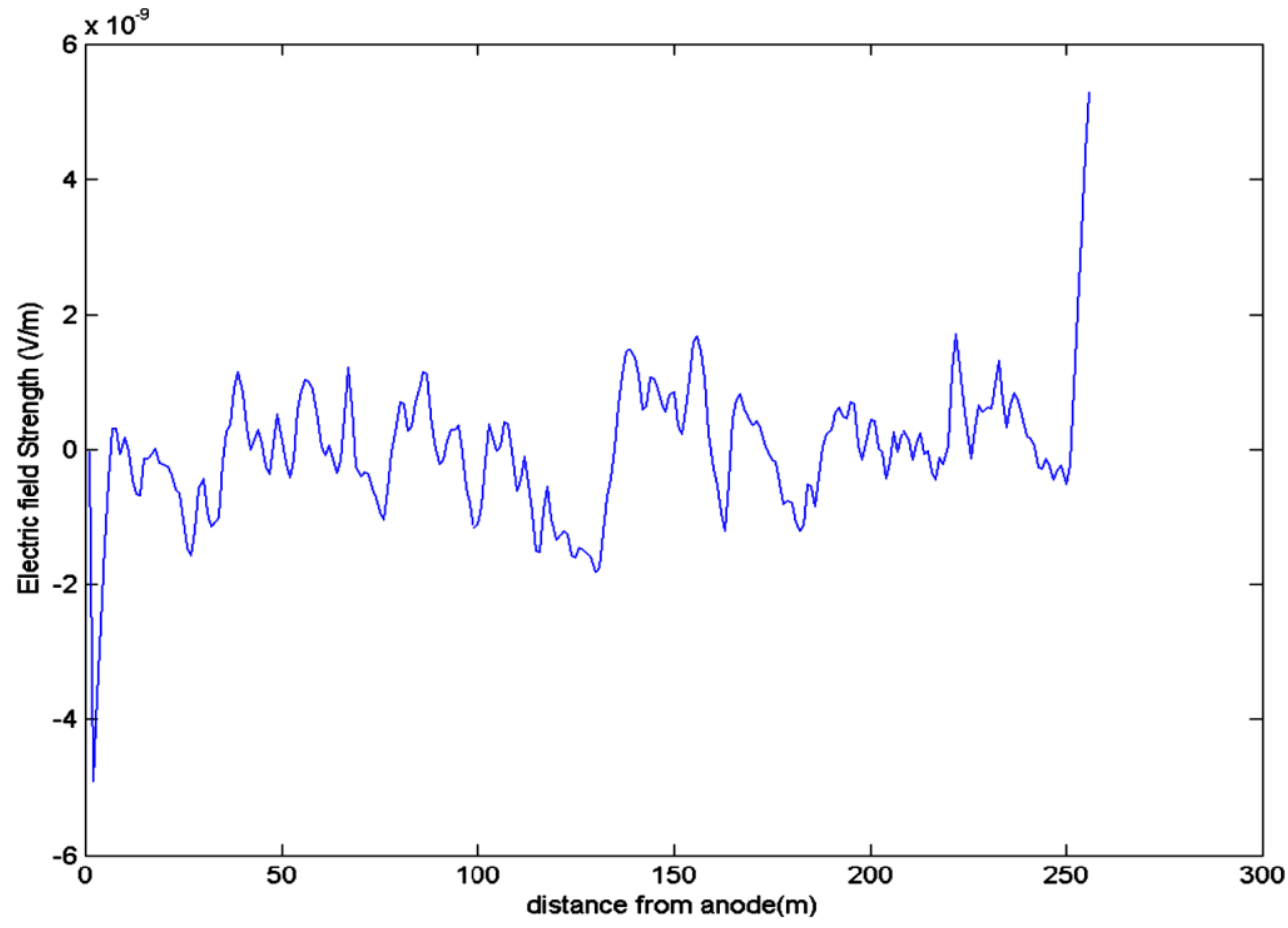

(b) $t=0.199 \mathrm{~s}, E_{\text {ext }}=0.5 \times 10^{-25} \mathrm{Vm}^{-1}$

Fig. 17. Electric field distribution over the distance at $(a) t=0.019 \mathrm{~s}$ and $(b) t=0.199 \mathrm{~s}$ without an external electric field and at $(c) t=0.019 \mathrm{~s}$ and $(d) t=0.199 \mathrm{~s}$. with external electric field. 


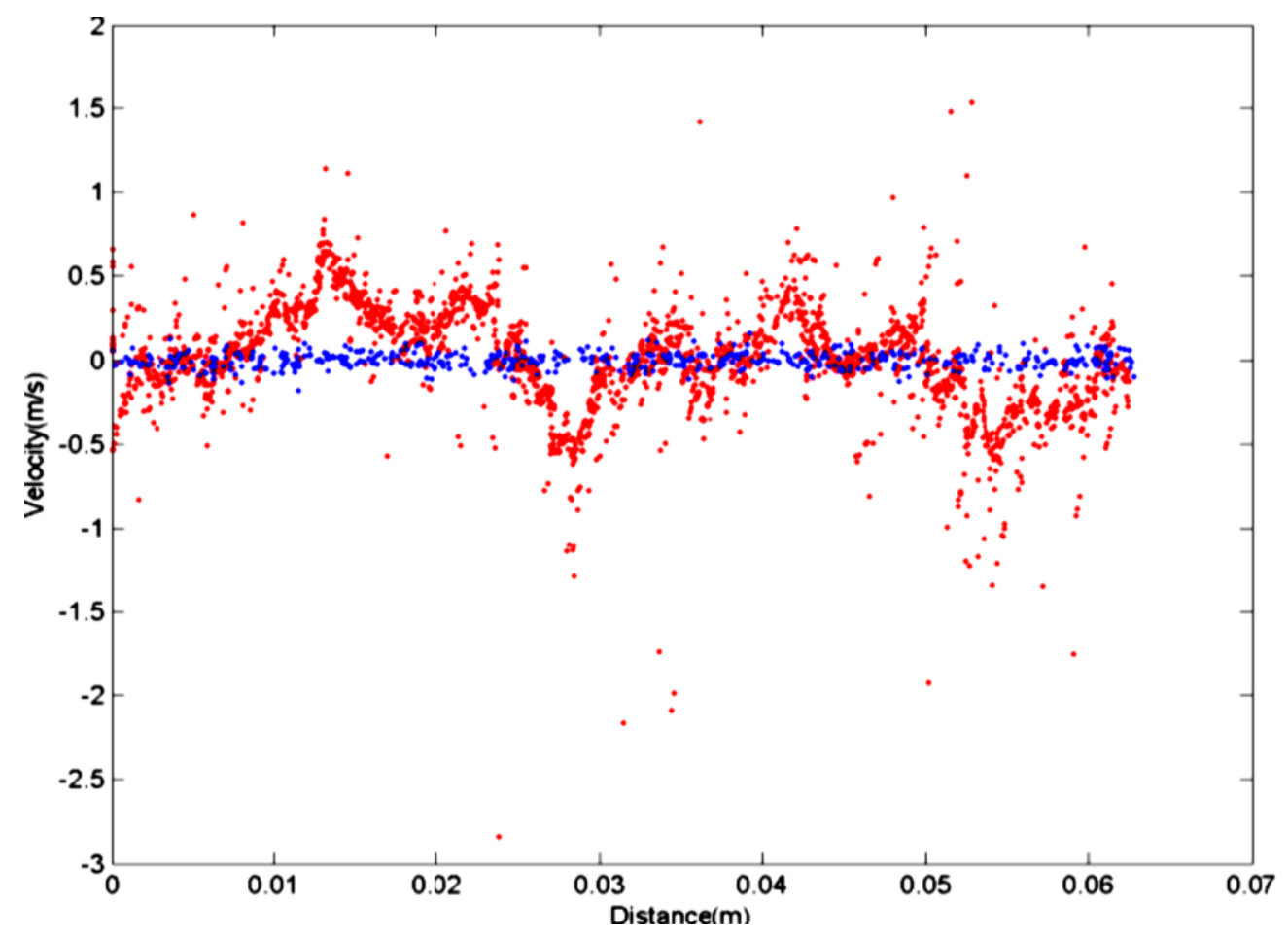

(a) $t=0.019 \mathrm{~s}$

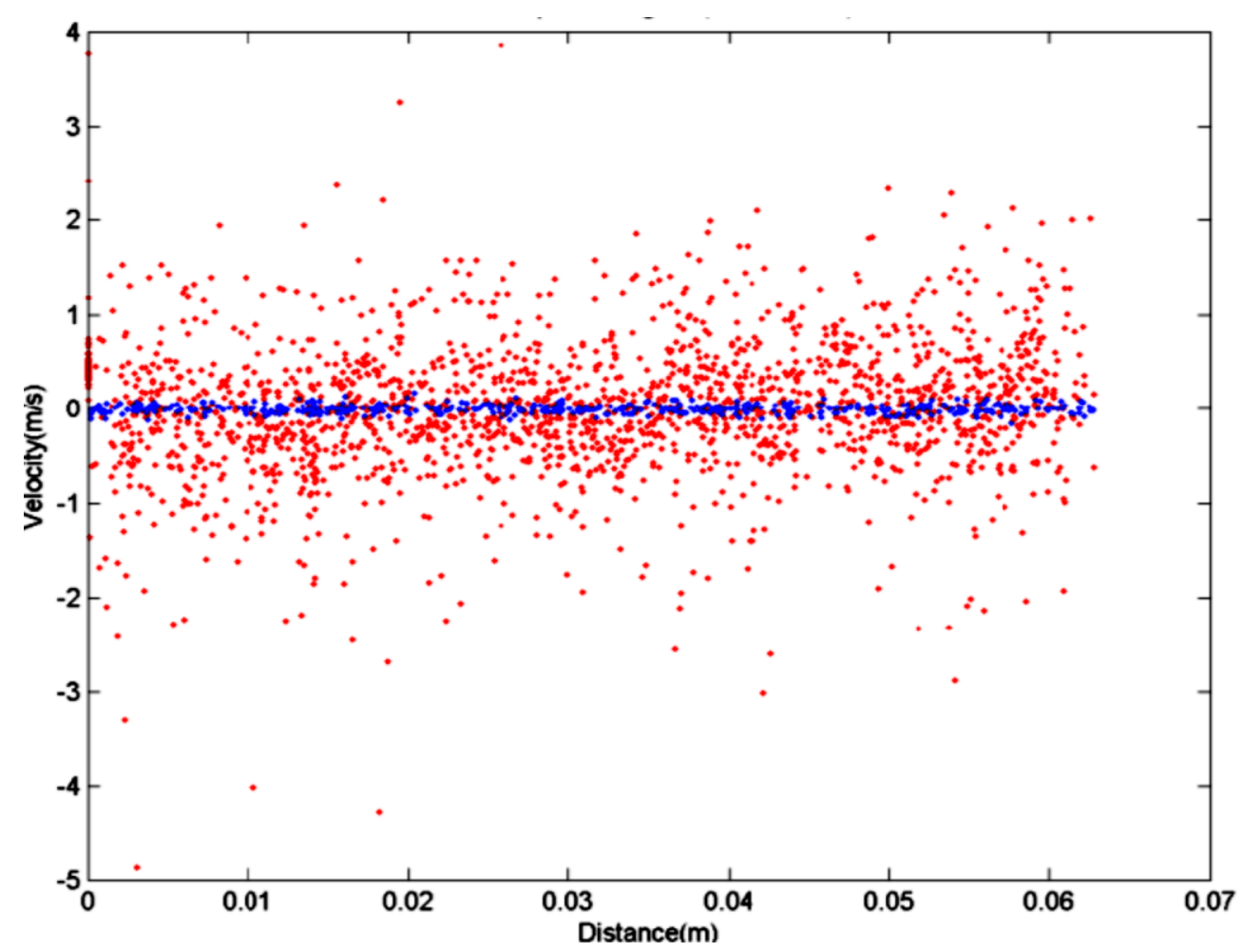

(b) $t=0.199 \mathrm{~s}$

Fig. 18. Phase Space diagrams of the particles inside the plasma for diode at $(a) t=0.019 \mathrm{~s}$ and (b) $t=0.199 \mathrm{~s}$ without an electric field. 
The distribution of the particles of plasma filled diode with and without an external electric field was the same except for the time taken for stabilized. With positive electric field this time is much less than for the ideal case while for negative field it is much greater.

\section{CONCLUSIONS}

Two types of numerical models for plasma diode systems have been implemented and the system was investigated under different parameter conditions. For the numerical model based on the phenomenon of current density equilibrium at the plasma boundary a method for finding working ranges for electron number density with the variation of amplitude of the applied pulse and the A-C gap has been presented. The system will become stable with time when the system parameters are in their respective working range. The theoretical work to find the minimal electron density for a plasma diode system with the maximum amplitude of input voltage pulses in the A-C gap is an attempt to find working limits of the model. The developed model can be used to find the lower limit of range of the electron density in which the model will work properly by obeying physical rules of the plasma diode.

By means of the numerical model, developed using a particle-in-cell simulation technique, the behaviour of the distribution of electric field and potential field and the behaviour of the charged particles inside the plasma over time for different values of externally applied electric fields was investigated with the assumptions that the anode cathode gap was completely filled with plasma and the inter-particle collisions are negligible. The system became stable after few time steps and the time taken to make the system stable, depends on the intensity and polarization of the external electric field.

\section{References}

[1] A. Dunaevsky, Ya. E. Krasik, J. Felsteiner, S. Dorfman, J. Appl. Phys. 85 (1999) 8474.

[2] H. Gundel, J. Hańderek, H. Riege, J. Appl. Phys. 69 (1991) 975.

[3] Ya. E. Krasik, K. Chirko, A. Sayapin, J. Gleizer, A. Krokhmal, J. Felsteiner, J. Appl. Phys. 94 (2003) 5158 .

[4] A. Dunaevsky, Ya. E. Krasik, J. Felsteiner, A. Sternlieb, J. Appl. Phys. 90 (2001) 3689.

[5] Ya. E. Krasik, A. Dunaevsky, J. Felsteiner, J.App. Phys. 85 (11) (1999) 7946.

[6] B. B. Godfrey, Phys. Fluids 30 (1987)1553.

[7] H Schamel, V Maslov, Phys.Rev. Lett. 70(8) (1993) 1105.

[8] D. Li, J. Zhang, X. Chen, IEEE Trans on Plasma Science 36(1) (2008) 146.

[9] M. O. Terra, J. J Barroso, E. E. N. Macau, Physica A 283(1-2) (2000) 119-124.

[10] C. K. Birdsall, A. B. Langdon, Plasma Physics via Computer Simulation (Taylor \& Francis Group, 1985).

[11] S. Schnepp, E. Gjonaj, T. Weiland, Proc. of EPAC, WEPCH114 (2006) 2182-2184. 